\title{
CONNECTING FAR-INFRARED AND RADIO MORPHOLOGIES OF DISK GALAXIES: COSMIC-RAY ELECTRON DIFFUSION AFTER STAR FORMATION EPISODES
}

\author{
E. J. Murphy, ${ }^{1}$ G. Helou, ${ }^{2}$ J. D. P. Kenney, ${ }^{1}$ L. Armus,${ }^{3}$ and R. Braun ${ }^{4}$ \\ Received 2007 July 4; accepted 2008 January 30
}

\begin{abstract}
We present results on the ISM properties of 29 galaxies based on a comparison of Spitzer far-infrared and Westerbork Synthesis Radio Telescope radio continuum imagery. Of these 29 galaxies, 18 are close enough to resolve at $\lesssim 1 \mathrm{kpc}$ scales at $70 \mu \mathrm{m}$ and $22 \mathrm{~cm}$. We extend the approach of our earlier work of smoothing infrared images to approximate cosmic-ray (CR) electron spreading and thus largely reproduce the appearance of radio images. Using a wavelet analysis, we decompose each $70 \mu \mathrm{m}$ image into one component containing the star-forming structures and a second one for the diffuse disk. The components are smoothed separately, and their combination compared to a free-free corrected $22 \mathrm{~cm}$ radio image; the scale lengths are then varied to best match the radio and smoothed infrared images. We find that late-type spirals having high amounts of ongoing star formation benefit most from the two-component method. We also find that the disk component dominates for galaxies having low star formation activity, whereas the structure component dominates at high star formation activity. We propose that this result arises from an age effect rather than from differences in CR electron diffusion due to varying ISM parameters. The bulk of the CR electron population in actively star-forming galaxies is significantly younger than that in less active galaxies due to recent episodes of enhanced star formation; these galaxies are observed within $\sim 10^{8} \mathrm{yr}$ since the onset of the most recent star formation episode. The sample irregulars have anomalously low best-fit scale lengths for their surface brightnesses compared to the rest of the sample spirals, which we attribute to enhanced CR electron escape.
\end{abstract}

Subject headings: cosmic rays — infrared: galaxies — radio continuum: galaxies

Online material: color figures

\section{INTRODUCTION}

The interstellar medium (ISM) is a complex environment comprised of a diverse mix of extremely tenuous matter (by terrestrial standards) spanning a range of energetic states. Atomic, ionized, and molecular material make up a series of gaseous (thermal) phases usually categorized by their temperature and density; combinations of these components are used to define the so-called two- and three-phase models of the ISM and variations thereof (e.g., McKee 1995 and references therein); however, this is not the entire picture.

There is an additional phase of the ISM that is often overlooked due to the difficulties associated with making direct observations of its constituents. This is the relativistic (nonthermal) phase, which is made up of relativistic charged particles known as cosmic rays (CRs) and magnetic fields. The CRs within galaxies have an energy density comparable to that of the gaseous phases (e.g., Boulares \& Cox 1990). They fill up the entire volume of galaxies and are important sources of heating and ionization of the ISM, although characterizing their propagation remains an ongoing astrophysical problem (for a review see Strong et al. 2007).

The relativistic components of the ISM are important dynamically and may play a significant role in the regulation of star formation during the formation and evolution of galaxies (e.g., Ferrière 2001; Cox 2005 and references therein). Magnetic fields both help to support interstellar matter against a galaxy's gravi-

\footnotetext{
1 Department of Astronomy, Yale University, New Haven, CT 06520-8101; murphy@astro.yale.edu.

2 California Institute of Technology, Pasadena, CA 91125.

3 Spitzer Science Center, California Institute of Technology, Pasadena, CA 91125.

4 ASTRON, 7990 AA Dwingeloo, Netherlands.
}

tational potential and confine CRs to galaxy disks; thus, magnetic fields and CRs take part in the hydrostatic balance and stability of the ISM while possibly determining the properties of gas spiral arms (Beck 2007) and even aiding in the triggering of star formation (Mouschovias et al. 1974; Elmegreen 1982). For sufficiently large CR pressures Parker instabilities (Parker 1966) can create breaches in magnetic disks, allowing CRs and interstellar material to freely stream into intergalactic space.

To date, most of our knowledge about the relativistic ISM outside of the Galaxy has been obtained indirectly through the detection of synchrotron emission via multifrequency radio observations (e.g., Duric 1991; Dahlem et al. 1995; Lisenfeld et al. 1996a; Irwin et al. 1999; Beck 2005). Synchrotron emission arises from $\mathrm{CR}$ electron energy losses as these particles are accelerated in the magnetic fields of galaxies. Although the energy density in CR electrons is only $\sim 1 \%$ of that for CR nuclei, the similarity between the spatial distributions of gamma-ray and synchrotron emission within the Galaxy suggests that CR electrons and CR nuclei are fairly well mixed on the scales of a few hundred parsecs (e.g., Haslam et al. 1982; Bloemen et al. 1986; Webber 1991). The spatial distribution of a galaxy's synchrotron emission is a function of a galaxy's CR electron and magnetic field distributions. Thus, radio synchrotron maps provide only limited insight on the source distribution of the CR electrons, as well as the distances the particles may have traveled before ending up in their current location of emission.

Massive stars $\left(\gtrsim 8 M_{\odot}\right)$ are the progenitors of supernovae (SNe) whose remnants (SNRs), through the process of diffusive shock acceleration (Bell 1978; Blandford \& Ostriker 1978), appear to be the main acceleration sites of $\mathrm{CR}$ electrons responsible for a galaxy's observed synchrotron emission. These same young massive stars are often the primary sources for dust heating as they emit photons that are reradiated at far-infrared (FIR) wavelengths. 
This shared origin between the FIR and radio emission of galaxies is thought to be the foundation for the observed FIR-radio correlation among (e.g., de Jong et al. 1985; Helou et al. 1985; Niklas 1997; Niklas \& Beck 1997; Yun et al. 2001) and within galaxies (e.g., Beck \& Golla 1988; Xu et al. 1992; Marsh \& Helou 1995; Hoernes et al. 1998; Hippelein et al. 2003; Murphy et al. 2006a, hereafter M06a; Hughes et al. 2006).

Coupling the shared origin of a galaxy's FIR and radio emission with the fact that the mean free path of dust-heating photons $(\sim 100 \mathrm{pc})$ is significantly shorter than the expected diffusion length of CR electrons ( $\sim 1-2 \mathrm{kpc}$ ) led Bicay \& Helou (1990) to conjecture that the radio image of a galaxy should resemble a smoothed version of its infrared image. Consequently, it appears that the close spatial correlation between the FIR and radio continuum emission within galaxies can be used to characterize the propagation history of CR electrons. This prescription has been shown to hold for galaxies observed at the "superresolution" $\left(\lesssim 1^{\prime}\right)$ of IRAS HIRES data (Marsh \& Helou 1998) and, more recently, for high-resolution $\left(\sim 18^{\prime \prime}\right)$ Spitzer $70 \mu \mathrm{m}$ imaging (M06a). This phenomenology has been further corroborated on scales $\gtrsim 50$ pc by Hughes et al. (2006), who find that synchrotron halos around individual star-forming regions are more extended than FIR-emitting regions within the Large Magellanic Cloud.

Murphy et al. (2006b, hereafter M06b) recently studied how the spatial distributions of a galaxy's FIR and radio emission vary as a function of the intensity of star formation. They concluded that CR electrons are, on average, younger and closer to their place of origin within galaxies having higher amounts of star formation activity compared with more quiescent galaxies. Using a wavelet-based image decomposition, we extend this work by attempting to characterize separately CR electron populations associated with a galaxy's diffuse disk and its star-forming complexes. We carry out this study for a sample of galaxies observed as part of the Spitzer Infrared Nearby Galaxies Survey (SINGS; Kennicutt et al. 2003) and the Westerbork Synthesis Radio Telescope (WSRT-SINGS; Braun et al. 2007) for which we have the spatial resolution to resolve physical scales $<1 \mathrm{kpc}$.

The paper is organized as follows: The galaxy sample is defined in $\S 2$, while the observations and data reduction techniques are discussed in $\S 3$. In $\S 4$ we present and discuss a correlation analysis between FIR/radio ratios and various other physical quantities on subkiloparsec scales within galaxies; this section leads us to confirm quantitatively that our favored phenomenological model is the best description for the FIR-radio correlation within galaxies. In $\S 5$ we describe our wavelet-based, twocomponent image-smearing analysis; the corresponding results are then presented in $\S 6$ and their physical implications are discussed in $\S 7$. We briefly discuss outstanding issues and future prospects in $\oint 8$ and summarize our results and conclusions in $\S 9$.

\section{GALAXY SAMPLE}

We present FIR and radio continuum imaging for 29 galaxies included in the SINGS (Kennicutt et al. 2003) sample. The SINGS sample consists of 75 normal galaxies at distances $\lesssim 30 \mathrm{Mpc}$. These objects were chosen to span a range of Hubble types (from irregulars to elliptical), as well as exhibit a large range in star formation rates (SFRs; $<0.001$ to $>10 M_{\odot} \mathrm{yr}^{-1}$ ), FIR/optical ratios $\left(\sim 10^{3}\right)$, and luminosity $\left(\sim 10^{5}\right)$; see Kennicutt et al. $(2003)$ for a more detailed description of the SINGS sample. The WSRT-SINGS subsample of 29 galaxies was then defined by choosing those SINGS galaxies that could be observed with
WSRT (i.e., north of declination $12.5^{\circ}$ ) and have $D_{25}>5^{\prime}$; these criteria ensured that the angular resolution across each target would be acceptable to allow for a resolved study of each galaxy disk. These 29 galaxies also span a similar range in physical parameters as the parent SINGS sample. See Braun et al. (2007) for a more detailed description of the WSRT-SINGS project.

In Table 1 we give basic data for each galaxy in the sample. Galaxy diameters $\left(D_{25}\right)$ and position angles were taken from the Third Reference Catalog of Bright Galaxies (RC3; de Vaucouleurs et al. 1991). We calculate inclinations using the method described by Dale et al. (1997) such that

$$
\cos ^{2} i=\frac{(b / a)^{2}-(b / a)_{\mathrm{int}}^{2}}{1-(b / a)_{\mathrm{int}}^{2}}
$$

where $a$ and $b$ are the observed semimajor and semiminor axes, respectively, and the disks are oblate spheroids with an intrinsic axial ratio $(b / a)_{\mathrm{int}} \simeq 0.2$ for morphological types earlier than Sbc and $(b / a)_{\text {int }} \simeq 0.13$ otherwise. Galaxy distances were taken from the literature with preference given to direct measurements (see Table 1). For those galaxies where direct measurements were not found we use flow-corrected estimates (Masters 2005). Of these 29 galaxies, 18 have distances less than $11.5 \mathrm{Mpc}$; these galaxies are resolved by the $70 \mu \mathrm{m}$ Spitzer beam at spatial scales $\lesssim 1 \mathrm{kpc}$. Due to the high spatial resolution for which we can examine the FIR-radio correlation within these galaxies, they are the focus of the present analysis.

\section{OBSERVATIONS AND DATA REDUCTION}

\subsection{Spitzer Images}

Observations at 24, 70, and $160 \mu \mathrm{m}$ were obtained using the Multiband Imaging Photometer for Spitzer (MIPS; Rieke et al. 2004) as part of the SINGS legacy science program. A detailed description of the SINGS observational strategy can be found in Kennicutt et al. (2003). The MIPS data were processed using the MIPS Data Analysis Tool (DAT; Gordon et al. 2005) and included in the SINGS data release 4 (DR4). Additional steps beyond the standard reduction procedure of the MIPS DAT are described in M06a. The full width at half-maximum (FWHM) of the MIPS 24, 70, and $160 \mu \mathrm{m}$ point-spread functions (PSFs) is $5.7^{\prime \prime}, 17^{\prime \prime}$, and $38^{\prime \prime}$, respectively. The mean rms noise values measured for the 24, 70, and $160 \mu \mathrm{m}$ maps are $\sim 0.048,0.447$, and $0.574 \mathrm{MJy} \mathrm{sr}^{-1}$, respectively. SINGS DR4 has improved the final calibration uncertainties to $\sim 4 \%, \sim 7 \%$, and $\sim 12 \%$ at 24 , 70 , and $160 \mu \mathrm{m}$, respectively. Some artifacts in the MIPS images have also been corrected for in DR4. In the $70 \mu \mathrm{m}$ data, very high surface brightness regions are known to be affected by nonlinearities in the calibration. The galaxy most strongly affected is NGC 3627 , where only $\sim 5 \%$ of the area may be miscalibrated.

\subsection{Radio Continuum Images}

Radio continuum imaging at $22 \mathrm{~cm}$ was performed using the WSRT as part of the WSRT-SINGS survey. A complete description of the radio observations and image processing steps can be found in Braun et al. (2007). To mitigate significant flux loss at low spatial frequencies (i.e., the zero-spacing problem), observations were taken using a configuration with particularly good sampling at short baselines. The WSRT data were CLEANed and self-calibrated using an imaging pipeline based on the 
TABLE 1

Basic Galaxy Data

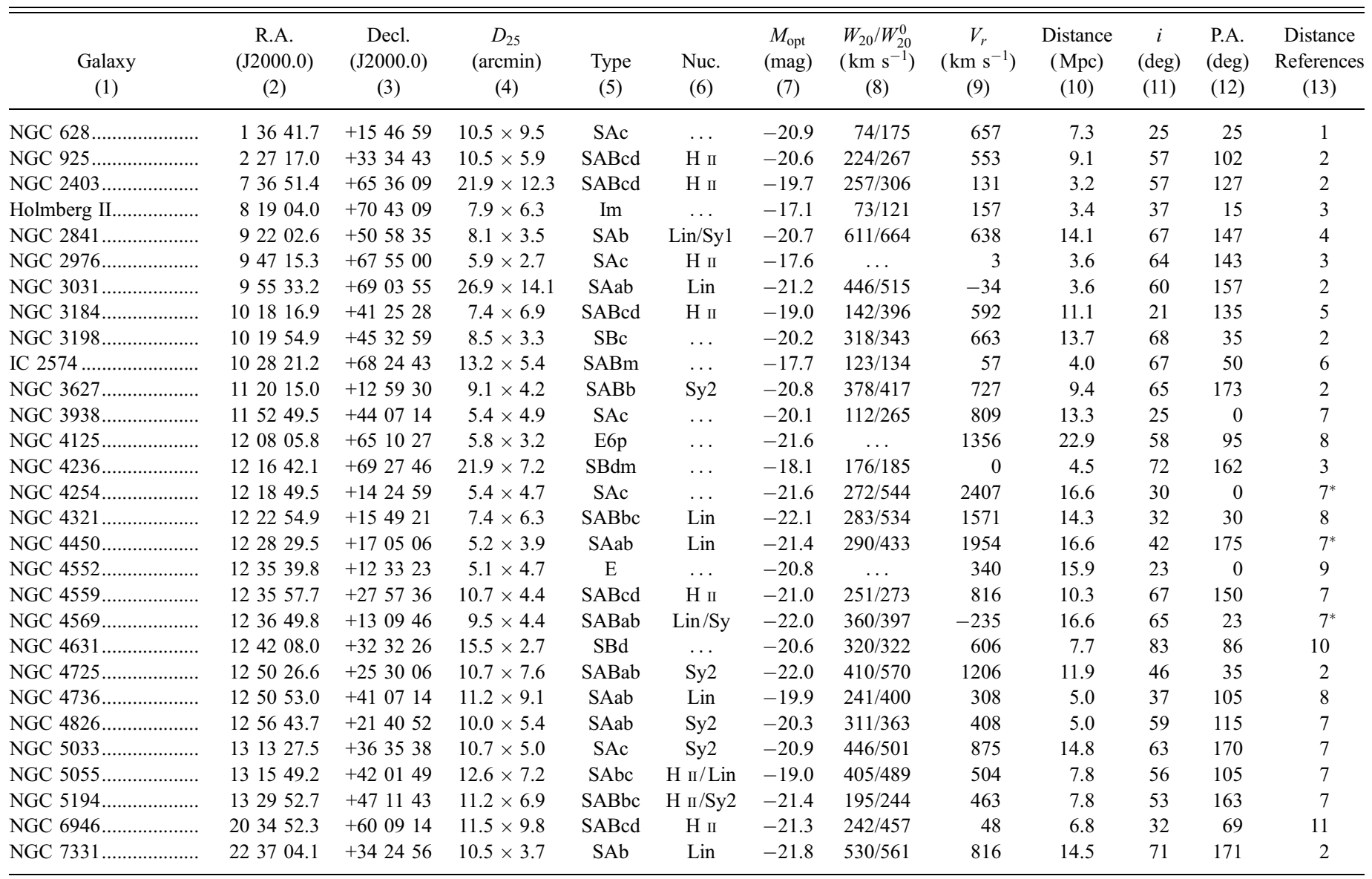

Notes._-Units of right ascension are hours, minutes, and seconds, and units of declination are degrees, arcminutes, and arcseconds. Col. (1): ID. Col. (2): Right ascension in the J2000.0 epoch. Col. (3): Declination in the J2000.0 epoch. Col. (4): Major- and minor-axis diameters. Col. (5): RC3 type. Col. (6): Nuclear type: H II = $\mathrm{H}$ II region; Lin =LINER; Sy = Seyfert $(1,2)$. Col. (7): Absolute $R$ magnitude, when available; otherwise, from the $V$ or $B$ band. Col. (8): Observed/inclination-corrected $21 \mathrm{~cm}$ neutral hydrogen line width at $20 \%$ of maximum intensity, taken from Tully (1988) or RC3. Col. (9): Heliocentric velocity. Col. (10): Distance. Col. (11): Inclination. Col. (12): Position angle. Col. (13): Distance references.

References.-(1) Shapley et al. 2001; (2) Freedman et al. 2001; (3) Karachentsev et al. 2002; (4) Macri et al. 2001; (5) Leonard et al. 2002; (6) Karachentsev et al. 2003; (7) Masters 2005; an asterisk indicates distance set to Virgo Cluster center; (8) Tonry et al. 2001; (9) Ferrarese et al. 2000; (10) Seth et al. 2005; (11) Karachentsev et al. 2000.

MIRIAD package. The final CLEAN maps were restored with $18^{\prime \prime}$ FWHM circular Gaussian beams. The intrinsic FWHM of the radio beams is approximately $11^{\prime \prime}$ east-west by $11 / \sin \delta^{\prime \prime}$ north-south at $1.4 \mathrm{GHz}$ and scales as wavelength, where $\delta$ is the source declination. Total intensity calibration of the data was performed in the AIPS package, and the flux density calibration of the radio maps is better than 5\%. In the case of NGC 3031 , which suffers from a combination of low extended surface brightness and confusion problems due to the nearby starburst galaxy NGC 3034, a $20 \mathrm{~cm}$ map was created via a variance-weighted average of both 22 and $18 \mathrm{~cm}$ data in order to obtain high-quality data. Accordingly, the flux density values of NGC 3031 were scaled to what is expected at $22 \mathrm{~cm}$ assuming a mean spectral index of -0.8 to allow for proper comparison with the rest of the sample. The mean rms noise in the radio maps is $\sim 4.4 \times$ $10^{-3} \mathrm{MJy} \mathrm{sr}^{-1}$.

\subsection{Ancillary Data}

Images in the $K_{s}$ band were taken from the Two Micron All Sky Survey (2MASS) Large Galaxy Atlas (LGA; Jarrett et al. 2003). The typical FWHM of PSFs for these images is $\sim 2.5^{\prime \prime}$.
Ultraviolet images were obtained by the Galaxy Evolution Explorer (GALEX) at 1528 and $2271 \AA$ as part of the GALEX Atlas of Nearby Galaxies (NGS; Gil de Paz et al. 2007). These galaxies, including nearly all of the SINGS sample, were observed with relatively deep integration times $(\sim 1.5 \mathrm{ks})$. With an angular resolution of $4^{\prime \prime}-6^{\prime \prime}$, the GALEX images are well matched to Spitzer $24 \mu \mathrm{m}$ imaging and more resolved than the Sptizer 70 and $160 \mu \mathrm{m}$ data. For a more detailed description of the GALEX observations see Gil de Paz et al. (2007). The only galaxy for which we use GALEX data that were not obtained as part of the NGS is NGC 4725; these data were obtained as part of the Allsky Imaging Survey (AIS; Martin et al. 2005) and have much shorter effective exposure times $(\sim 0.1 \mathrm{ks})$. Galaxies in the present sample that have not yet been observed by GALEX, or for which the data are not publicly available, include NGC 3184 and NGC 6946.

\subsection{Image Registration and Resolution Matching}

We match the resolution of the MIPS and radio images using Gaussian PSFs rather than the MIPS PSFs, which suffer from significant power in their sidelobes. After cropping each set of 


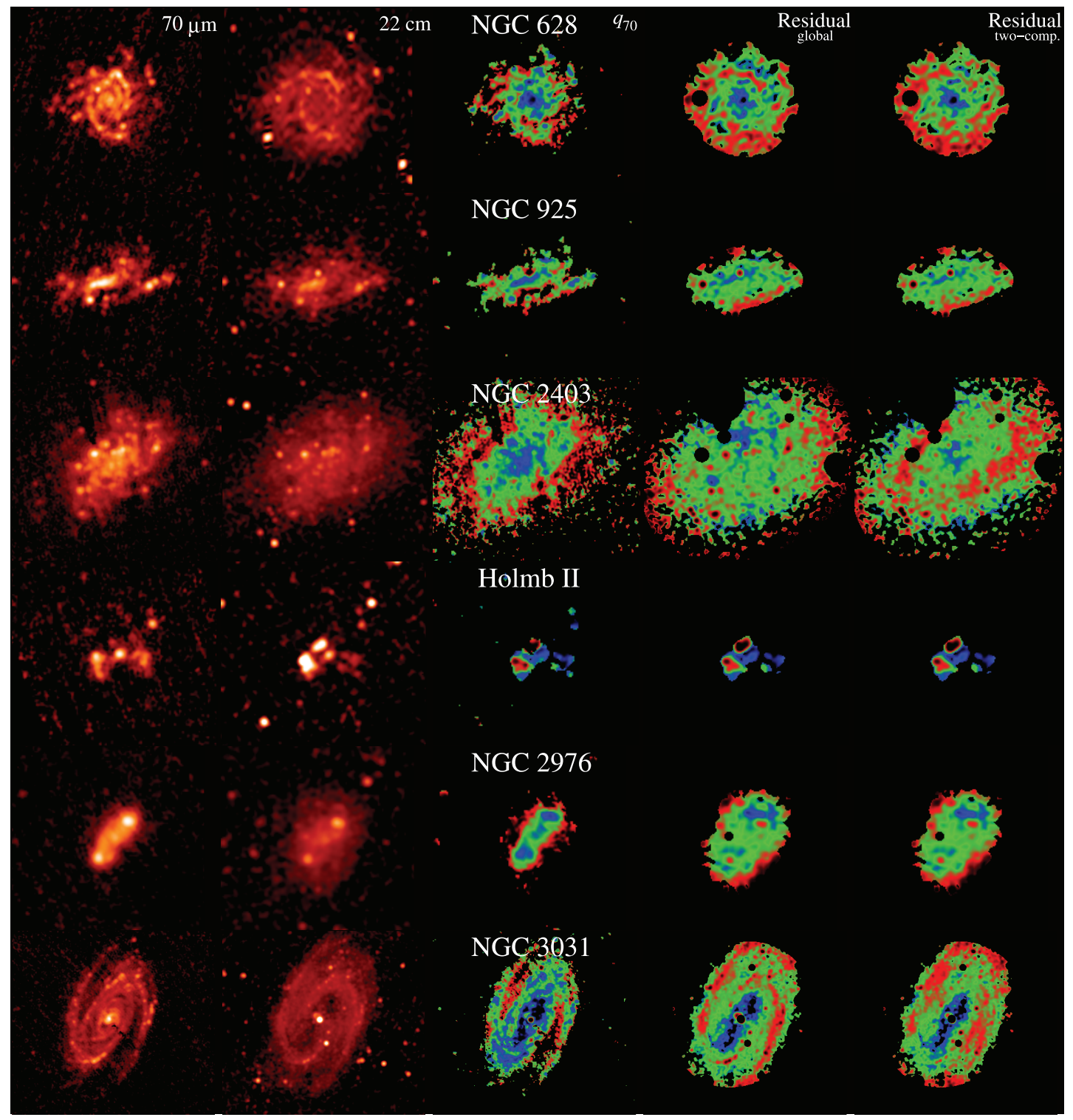

FIG. 1.-Each galaxy's $70 \mu \mathrm{m}$ and $22 \mathrm{~cm}$ images are displayed in the first and second columns, respectively. These images are shown using a logarithmic stretch ranging from the $1 \sigma \mathrm{rms}$ level of the background to the maximum surface brightness of the galaxy. A normalized $q_{70}$ map and the residual images between the $22 \mathrm{~cm}$ and $70 \mu \mathrm{m}$ images, smoothed with the best-fit single smearing kernel and best-fit disk and structure smearing kernels, are given in the third, fourth, and fifth columns, respectively. The residual map definition is given in $\S 5.4$. The stretch of each of these three maps runs from -0.75 to 0.75 dex; red and blue colors correspond to radio and infrared excesses in the residuals, respectively, while green corresponds to residuals $\sim 0$. Regions removed for the residual calculations (e.g., background radio sources) appear as dark, circular holes in a few of the residual maps.

galaxy images to a common field of view and regridding them to a common pixel scale, we divide the Fourier transform (FT) of the final MIPS images at each band by the FT of a model of the corresponding MIPS PSF. Next, this quotient is multiplied by the FT of a Gaussian PSF matching that of the CLEAN beam used to restore the corresponding radio images. The final product was then taken back into the image plane where we checked to ensure that flux was conserved throughout the resolution matching procedure. The resolution-matched $70 \mu \mathrm{m}$ and $22 \mathrm{~cm}$ maps for each galaxy are presented in the first and second columns of Figure 1, respectively.

The 2MASS $K_{s}$ band images were, likewise, registered, convolved, and regridded to match the MIPS $70 \mu \mathrm{m}$ data. The same was done for the GALEX far-UV (FUV; $1528 \AA$ ) and near-UV (NUV; $2271 \AA$ ) images after each was first corrected for Milky
Way extinction using the Galactic color excesses of Schlegel et al. (1998). The FUV and NUV images were then added to create a single GALEX "total UV" image for each galaxy, which we have used in the present analysis.

Once the PSF matching of the registered $70 \mu \mathrm{m}$ and $22 \mathrm{~cm}$ maps was completed, $q_{70}$ maps were constructed where

$$
q_{70} \equiv \log \left[\frac{f_{\nu}(70 \mu \mathrm{m})(\mathrm{Jy})}{S_{\nu}(22 \mathrm{~cm})(\mathrm{Jy})}\right] .
$$

\subsection{Global Flux Densities}

Global flux densities at $22 \mathrm{~cm}$ and at each MIPS band were measured by summing the flux density at each wavelength within elliptical apertures. Any identifiable flux contributions 


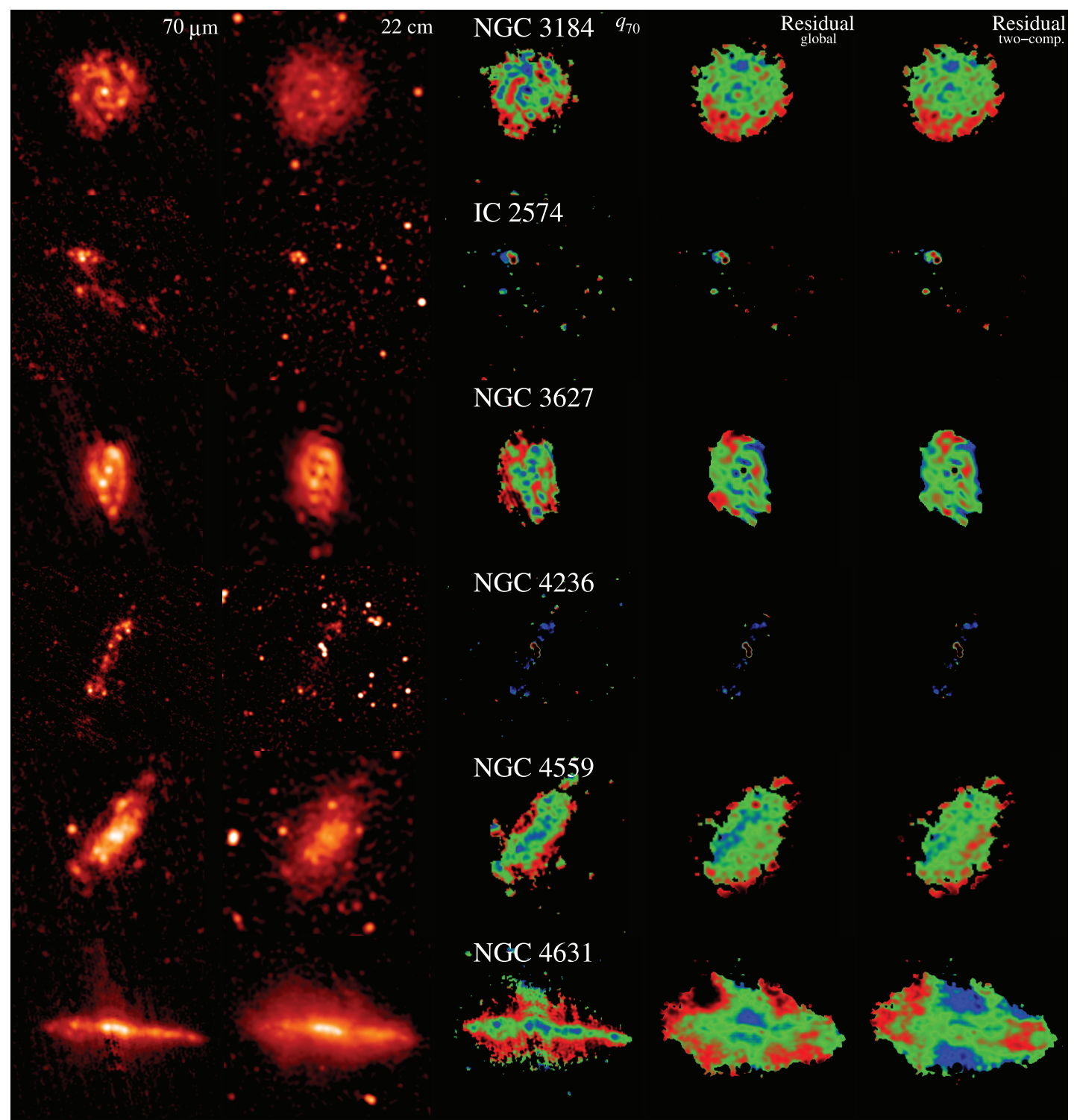

FIG. 1-Continued

from background sources were masked out in the measurements. In the case of NGC 5194, emission from its companion galaxy was also excluded in the global flux density measurements. These MIPS and radio flux densities agree with those presented in Dale et al. (2007) and Braun et al. (2007) (within errors). We therefore choose to adopt their values for consistency purposes. The only notable exceptions are the $22 \mathrm{~cm}$ flux densities of NGC 3031 and NGC 5194. Our radio flux density value for NGC 3031 was measured using the global aperture defined by Dale et al. (2007) and our averaged $20 \mathrm{~cm}$ map (see $\S 3.2$ ). We then scaled this flux density to what is expected at $22 \mathrm{~cm}$ assuming a mean spectral index of -0.8 to allow for proper comparison with the rest of the sample. Unlike Braun et al. (2007), we mask out flux contributions from NGC 5195 when measuring the $22 \mathrm{~cm}$ flux density of NGC 5194. The monochromatic $22 \mathrm{~cm}, 24 \mu \mathrm{m}, 70 \mu \mathrm{m}$, and $160 \mu \mathrm{m}$ flux densities for each sample galaxy are listed in Table 2.

Using a weighted combination of the MIPS flux densities, we compute estimates of the total-infrared (TIR; 3-1100 $\mu \mathrm{m}$ ) flux of each galaxy according to equation (4) of Dale \& Helou (2002). We also compute an estimate of the IRAS-based FIR $(42-122 \mu \mathrm{m})$ fractions using the same spectral energy distribution (SED) models of Dale \& Helou (2002) such that

$$
\frac{F_{\mathrm{FIR}}}{F_{\mathrm{TIR}}}=\sum_{i=0}^{3} \xi_{i} \log \left[\frac{f_{\nu}(70 \mu \mathrm{m})}{f_{\nu}(160 \mu \mathrm{m})}\right]^{i}
$$

where $\left[\xi_{0}, \xi_{1}, \xi_{2}, \xi_{3}\right]=[0.5158,0.1619,-0.3158,-0.1418]$ and $-0.65 \leq \log f_{\nu}(70 \mu \mathrm{m}) / f_{\nu}(160 \mu \mathrm{m}) \leq 0.54$.

The TIR fluxes were then used to calculate global $q_{\mathrm{TIR}}$ ratios for each galaxy where

$$
q_{\mathrm{TIR}} \equiv \log \left(\frac{F_{\mathrm{TIR}}}{3.75 \times 10^{12} \mathrm{~W} \mathrm{~m}^{-2}}\right)-\log \left[\frac{S_{\nu}(22 \mathrm{~cm})}{\mathrm{W} \mathrm{m}^{-2} \mathrm{~Hz}^{-1}}\right] .
$$




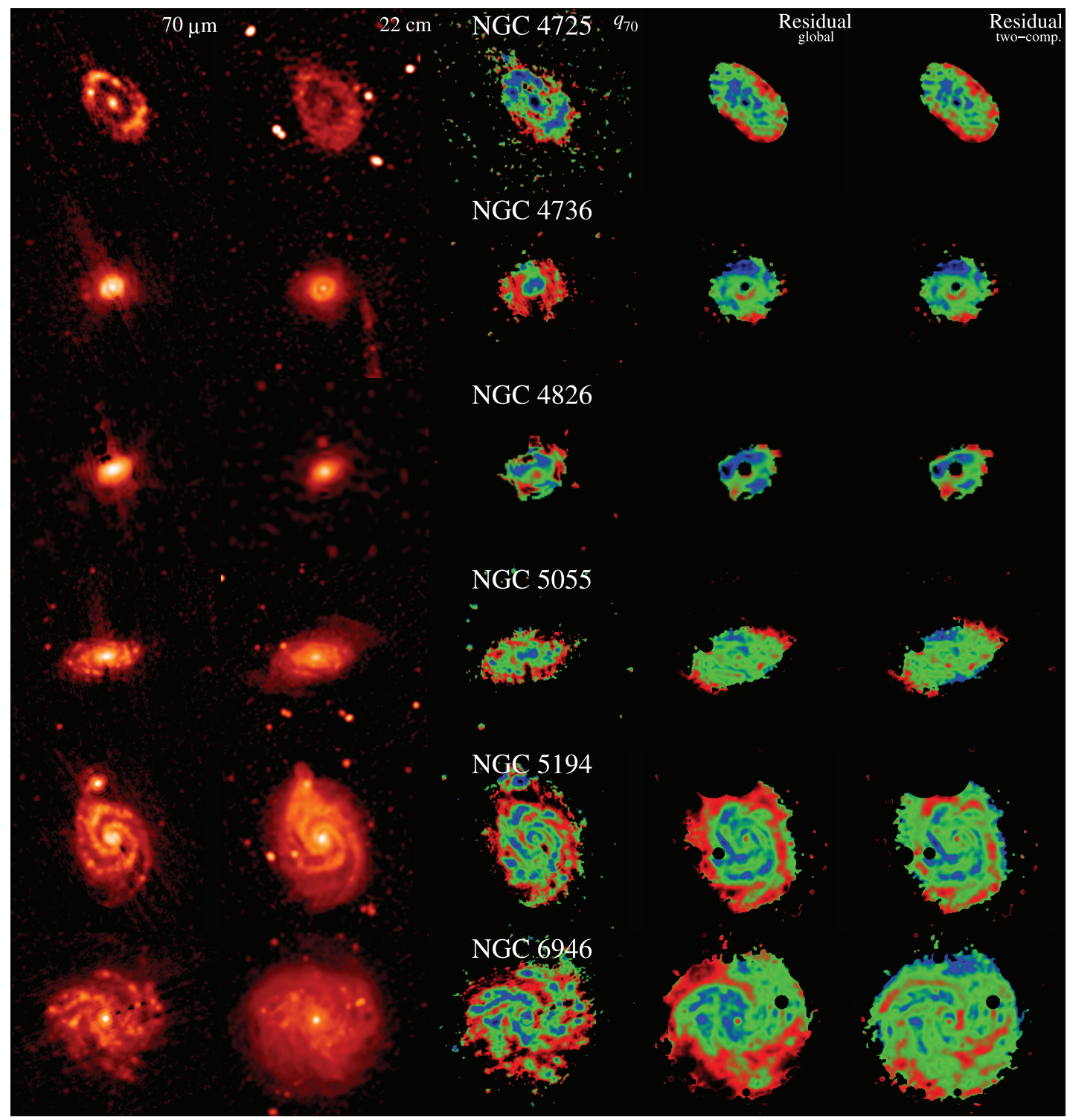

FIG. 1-Continued

The more commonly used $q$ of Helou et al. (1985), which we denote as $q_{\text {FIR }}$, is also calculated such that

$$
q_{\mathrm{FIR}} \equiv \log \left(\frac{F_{\mathrm{FIR}}}{3.75 \times 10^{12} \mathrm{~W} \mathrm{~m}^{-2}}\right)-\log \left[\frac{S_{\nu}(22 \mathrm{~cm})}{\mathrm{W} \mathrm{m}^{-2} \mathrm{~Hz}^{-1}}\right]
$$

The FIR and TIR fluxes, along with the associated $q_{\text {FIR }}$ and $q_{\mathrm{TIR}}$ ratios, are given in Table 2 .

\subsection{Radiation Field Energy Densities}

We compute the radiation field energy density $\left(U_{\text {rad }}\right)$ of each galaxy using its TIR surface brightness since this parameter is sensitive to the diffusion of CR electrons. This was done by first repeating the above image registration and resolution matching at each MIPS wave band using a $45^{\prime \prime}$ Gaussian beam (i.e., slightly larger than the FWHM of the native $160 \mu \mathrm{m}$ beam), creating TIR surface brightness maps for each galaxy using a weighted combination of the three MIPS bands according to equation (4) of Dale \& Helou (2002) on a pixel-by-pixel basis. The TIR lum- inosity $\left(L_{\mathrm{TIR}}\right)$ was then summed within elliptical apertures fitted to a galaxy's $1.4 \times 10^{-7} \mathrm{~W} \mathrm{~m}^{-2} \mathrm{sr}^{-1}$ isophotal radius; this radius corresponds to the maximum $3 \sigma$ rms value among the entire sample's TIR maps and was used to calculate the isophotal TIR diameters $D_{\text {TIR }}$ for each galaxy. We note that these luminosities will be less than those corresponding to the TIR fluxes given in Table 2 as those fluxes were measured within much larger apertures, effectively going into the noise of each MIPS map, as given by Dale et al. (2007). Using the deprojected area of each elliptical aperture, $A_{\mathrm{TIR}}=\pi\left(D_{\mathrm{TIR}} / 2\right)^{2}$, we calculate TIR surface brightnesses $\left(\Sigma_{\mathrm{TIR}}=L_{\mathrm{TIR}} / A_{\mathrm{TIR}}\right)$ along with estimates of $U_{\text {rad }}$ for radiation emitted near the surface of a semitransparent body such that

$$
U_{\mathrm{rad}} \approx \frac{2 \pi}{c} I_{\mathrm{bol}} \gtrsim \frac{L_{\mathrm{TIR}}}{2 A_{\mathrm{TIR}} c}\left(1+\sqrt{\frac{3.8 \times 10^{42}}{L_{\mathrm{TIR}}}}\right),
$$

where $I_{\text {bol }}$ is a galaxy's bolometric surface brightness, $c$ is the speed of light, and all quantities are given in cgs units. The parenthetical term in equation (6) provides a correction for nonabsorbed UV emission that was empirically derived by Bell 
TABLE 2

Global Flux Densities and Derived Parameters

\begin{tabular}{|c|c|c|c|c|c|c|c|c|}
\hline Galaxy & $\begin{array}{c}S_{\nu}(22 \mathrm{~cm}) \\
\quad(\mathrm{Jy})\end{array}$ & $\begin{array}{c}f_{\nu}(24 \mu \mathrm{m}) \\
(\mathrm{Jy})\end{array}$ & $\begin{array}{c}f_{\nu}(70 \mu \mathrm{m}) \\
(\mathrm{Jy})\end{array}$ & $\begin{array}{c}f_{\nu}(160 \mu \mathrm{m}) \\
(\mathrm{Jy})\end{array}$ & $\begin{array}{c}F_{\mathrm{FIR}} \\
\left(10^{-13} \mathrm{~W} \mathrm{~m}^{-2}\right)\end{array}$ & $\begin{array}{c}F_{\mathrm{TIR}} \\
\left(10^{-13} \mathrm{~W} \mathrm{~m}^{-2}\right)\end{array}$ & $q_{\mathrm{FIR}}$ & $q_{\mathrm{TIR}}$ \\
\hline NGC $628 \ldots \ldots \ldots \ldots \ldots \ldots$ & 0.200 & 3.19 & 34.78 & 126.2 & 17.36 & 49.39 & 2.36 & 2.82 \\
\hline 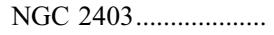 & 0.360 & 5.84 & 86.36 & 245.6 & 39.64 & 101.5 & 2.47 & 2.88 \\
\hline Holmberg II..................... & 0.005 & 0.20 & 3.67 & 4.46 & 1.36 & 2.73 & 2.82 & 3.12 \\
\hline 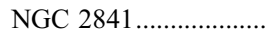 & 0.100 & 0.91 & 10.22 & 62.29 & 6.53 & 20.80 & 2.24 & 2.74 \\
\hline 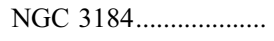 & 0.080 & 1.43 & 15.76 & 70.48 & 8.10 & 25.69 & 2.43 & 2.93 \\
\hline 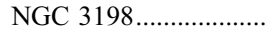 & 0.049 & 1.06 & 10.27 & 39.00 & 5.24 & 15.24 & 2.46 & 2.92 \\
\hline IC 2574 & 0.014 & 0.28 & 5.55 & 11.75 & 2.32 & 5.33 & 2.65 & 3.01 \\
\hline NGC $3627 \ldots \ldots \ldots \ldots \ldots$ & 0.500 & 7.42 & 92.63 & 230.2 & 42.24 & 102.8 & 2.35 & 2.74 \\
\hline 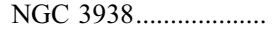 & 0.080 & 1.09 & 14.25 & 51.98 & 6.97 & 19.87 & 2.37 & 2.82 \\
\hline NGC $4125 \ldots \ldots \ldots \ldots \ldots$ & 0.002 & 0.079 & 1.11 & 1.77 & 0.46 & 0.96 & 2.81 & 3.13 \\
\hline NGC 4236...................... & 0.026 & 0.55 & 8.27 & 20.43 & 3.67 & 8.92 & 2.58 & 2.96 \\
\hline NGC $4559 \ldots \ldots \ldots \ldots$ & 0.110 & 1.12 & 16.89 & 54.15 & 7.93 & 21.35 & 2.28 & 2.71 \\
\hline NGC $4569 \ldots \ldots \ldots \ldots$ & 0.170 & 1.44 & 12.37 & 41.21 & 6.29 & 17.23 & 1.99 & 2.43 \\
\hline 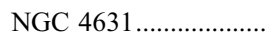 & 1.290 & 8.15 & 130.2 & 289.5 & 56.22 & 131.5 & 2.06 & 2.43 \\
\hline NGC $4725 \ldots \ldots \ldots \ldots$ & 0.100 & 0.86 & 8.85 & 59.91 & 6.17 & 19.66 & 2.22 & 2.72 \\
\hline 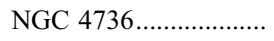 & 0.320 & 5.65 & 93.93 & 177.4 & 38.94 & 86.47 & 2.51 & 2.86 \\
\hline 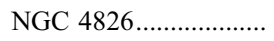 & 0.110 & 2.72 & 55.16 & 98.82 & 22.06 & 48.28 & 2.73 & 3.07 \\
\hline NGC 5033 & 0.240 & 1.97 & 28.81 & 91.07 & 13.54 & 36.21 & 2.18 & 2.60 \\
\hline NGC 5055 & 0.450 & 5.73 & 72.57 & 302.3 & 36.43 & 111.1 & 2.33 & 2.82 \\
\hline 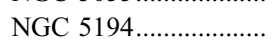 & $1.240^{\mathrm{b}}$ & 12.67 & 147.1 & 494.8 & 71.84 & 197.5 & 2.19 & 2.63 \\
\hline NGC 6946..................... & 1.700 & 20.37 & 207.2 & 502.8 & 97.10 & 234.1 & 2.18 & 2.56 \\
\hline NGC $7331 \ldots \ldots \ldots \ldots \ldots$ & 0.590 & 4.36 & 74.97 & 189.5 & 33.01 & 80.79 & 2.17 & 2.56 \\
\hline
\end{tabular}

Note.-Radio flux densities taken from Braun et al. (2007); MIPS flux densities taken from Dale et al. (2007).

a This flux density, measured using a $20 \mathrm{~cm}$ map, was scaled to the expected flux density at $22 \mathrm{~cm}$ assuming a spectral index of -0.8 .

${ }^{\mathrm{b}}$ Corrected for flux contributions from companion galaxy NGC 5195 .

(2003) using archived FIR and UV data for a sample of more than 200 galaxies.

Since $U_{\text {rad }}$ is likely proportional to the disk-averaged star formation activity within the normal star-forming galaxies considered here, we also express $U_{\text {rad }}$, for illustration purposes, as a star formation rate surface density, $\Sigma_{\mathrm{SFR}}$, again using Bell (2003). The TIR diameters along with values of $\Sigma_{\text {TIR }}$ and $U_{\text {rad }}$, expressed in units of $L_{\odot} \mathrm{kpc}^{-2}$ and ergs $\mathrm{cm}^{-3}$, respectively, are given in Table 3.

\section{CORRELATION ANALYSIS OF $q_{70}$}

M06a showed that the FIR/radio ratio is not constant within galaxies, exhibiting strong variation patterns in residual maps and a dispersion as small as the dispersion in the FIR/radio ratios among galaxies. Some of the variation in the FIR/radio ratios within galaxies was found to be related to both infrared surface brightness and radius. Using our larger sample, we confirm these results here, as well as expand the explored parameter space. We search for the dominant physical parameter related to the variations in the FIR/radio ratio within galaxies by plotting $q_{70}$ against a number of physical quantities and calculating the residual dispersion in each fit. This was done for galaxies sufficiently nearby that the resolution at $70 \mu \mathrm{m}$ is better than $1 \mathrm{kpc}$.

\section{1. $q_{70}$ Correlation Analysis within Galaxies}

We examine the relations between $q_{70}$ and (1) $24 \mu \mathrm{m}$ surface brightness, since it has been found to trace local massive star formation activity (e.g., Calzetti et al. 2005, 2007); (2) $K_{s}$-band surface brightness (tracer of stellar mass); (3) UV surface brightness (extinction-dependent tracer of local star formation); (4) galactocentric radius; (5) $24 \mu \mathrm{m} / 70 \mu \mathrm{m}$ surface brightness ratio (local heating of dust); and (6) $24 \mu \mathrm{m} / \mathrm{UV}$ surface brightness ratio (obscured star formation and permeability of ISM). Each of the UV, $K_{s}, 24 \mu \mathrm{m}, 70 \mu \mathrm{m}$, and $22 \mathrm{~cm}$ images was regridded with $1 \mathrm{kpc}^{2}$ pixels. Pixels in the regridded images of each galaxy were removed if they were either below the $3 \sigma$ level or associated with emission from nonrelated objects, such as background galaxies. Galaxies having $<10$ such resolution elements remaining were excluded in the analysis (i.e., Holmberg II and IC 2574).

An example of the $q_{70}$ scatter plots for each of the six tested parameters is given for NGC 628 in Figure 2. Each relation is fitted using an ordinary least-squares (OLS) regression of the form $q_{70}=m \log X+b$, where $X$ is one of the six parameters cited above, $m$ is the slope of the fit, and $b$ is the $y$-intercept of the fit. To quantitatively determine which of these six parameters best correlates with the FIR/radio ratio, we compute the residual dispersion about the regression line, denoted as $\sigma_{C}(X)$. Average $q_{70}$ ratios and the associated dispersions are given in Table 4 along with the residual dispersions for each parameter and galaxy; the means for the entire sample are also presented.

\subsection{Results of $q_{70}$ Correlation Analysis}

We find that the residual dispersion around the trend of $q_{70}$ versus $24 \mu \mathrm{m}$ surface brightness is, on average, $\sim 0.07 \mathrm{dex}$ 
TABLE 3

Radiation Field Energy Densities and Star Formation Rate Surface Densities

\begin{tabular}{|c|c|c|c|}
\hline Galaxy & $\begin{array}{l}D_{\mathrm{TIR}} \\
(\mathrm{kpc})\end{array}$ & $\begin{array}{c}\log \Sigma_{\mathrm{TIR}} \\
\left(L_{\odot} \mathrm{kpc}^{-2}\right)\end{array}$ & $\begin{array}{c}\log U_{\text {rad }}(\mathrm{TIR}+\mathrm{UV}) \\
\left(\mathrm{ergs} \mathrm{cm}^{-3}\right)\end{array}$ \\
\hline NGC $628 \ldots \ldots \ldots \ldots \ldots . .$. & 20.42 & 7.37 & -12.67 \\
\hline 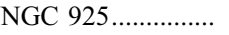 & 24.42 & 6.94 & -13.06 \\
\hline NGC $2403 \ldots \ldots \ldots \ldots . . .$. & 12.79 & 7.38 & -12.60 \\
\hline Holmberg II............. & 4.76 & 6.57 & -12.91 \\
\hline NGC $2841 \ldots \ldots \ldots \ldots . .$. & 30.69 & 7.21 & -12.86 \\
\hline NGC $2976 \ldots \ldots \ldots \ldots . . . .$. & 5.98 & 7.48 & -12.38 \\
\hline 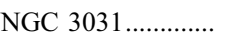 & 20.12 & 7.19 & -12.82 \\
\hline 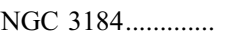 & 21.33 & 7.42 & -12.63 \\
\hline 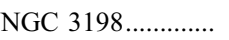 & 27.07 & 7.16 & -12.89 \\
\hline 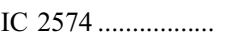 & 10.68 & 6.34 & -13.32 \\
\hline 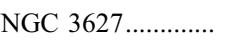 & 23.10 & 7.80 & -12.30 \\
\hline NGC $3938 \ldots \ldots \ldots \ldots$ & 20.85 & 7.48 & -12.57 \\
\hline NGC $4125 \ldots \ldots \ldots \ldots$ & 13.79 & 6.88 & -13.00 \\
\hline NGC $4236 \ldots \ldots \ldots \ldots$ & 15.50 & 6.33 & -13.44 \\
\hline NGC $4254 \ldots \ldots \ldots \ldots . . . .$. & 31.47 & 7.80 & -12.31 \\
\hline NGC $4321 \ldots \ldots \ldots \ldots . . . . .$. & 30.07 & 7.67 & -12.43 \\
\hline NGC $4450 \ldots \ldots \ldots \ldots . . . .$. & 20.17 & 7.11 & -12.89 \\
\hline NGC $4552 \ldots \ldots \ldots \ldots . .$. & 4.51 & 6.70 & -12.82 \\
\hline NGC $4559 \ldots \ldots \ldots \ldots . . . .$. & 24.52 & 7.14 & -12.89 \\
\hline 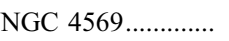 & 21.50 & 7.59 & -12.48 \\
\hline NGC $4631 \ldots \ldots \ldots \ldots . . . .$. & 27.57 & 7.58 & -12.51 \\
\hline NGC $4725 \ldots \ldots \ldots \ldots . . .$. & 30.59 & 7.01 & -13.03 \\
\hline 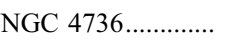 & 13.76 & 7.61 & -12.42 \\
\hline 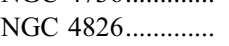 & 8.14 & 7.77 & -12.20 \\
\hline NGC $5033 \ldots \ldots \ldots \ldots . . . . .$. & 40.23 & 7.25 & -12.84 \\
\hline 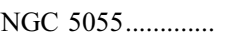 & 26.45 & 7.56 & -12.52 \\
\hline NGC 5194................ & 30.46 & 7.68 & -12.42 \\
\hline 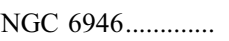 & 27.81 & 7.73 & -12.38 \\
\hline NGC $7331 \ldots \ldots \ldots \ldots . .$. & 44.81 & 7.47 & -12.64 \\
\hline
\end{tabular}

smaller than the dispersion in $q_{70}$; this is the largest improvement among all the parameters inspected. The average values of $\sigma_{C}\left[I_{\nu}\left(K_{s}\right)\right], \sigma_{C}\left[I_{\nu}(\mathrm{UV})\right], \sigma_{C}$ (radius), $\sigma_{C}\left[I_{\nu}(24 \mu \mathrm{m}) / I_{\nu}(70 \mu \mathrm{m})\right]$, and $\sigma_{C}\left[I_{\nu}(24 \mu \mathrm{m}) / I_{\nu}(\mathrm{UV})\right]$ are $\sim 0.03,0.02,0.04,0.05$, and 0.05 dex larger, respectively, than the average value of $\sigma_{C}\left[I_{\nu}(24 \mu \mathrm{m})\right]$, suggesting that the FIR/radio ratio is most strongly correlated on $24 \mu \mathrm{m}$ surface brightness. A galaxy's $24 \mu \mathrm{m}$ surface brightness appears to be a good proxy for extinction-corrected $\mathrm{Pa} \alpha$ and therefore the amount of ongoing massive star formation activity within a galaxy (e.g., Calzetti et al. 2005); however, we note that a combination of $24 \mu \mathrm{m}$ and $\mathrm{H} \alpha$ emission, to account for unobscured star formation, has been found to be a better proxy than $24 \mu \mathrm{m}$ emission alone (i.e., Calzetti et al. 2007; Kennicutt et al. 2007). The fact that the residual dispersion between $q_{70}$ and UV surface brightness is nearly as small as the residual dispersion around the trend of $q_{70}$ and $24 \mu \mathrm{m}$ surface brightness suggests that, at least at kiloparsec scales, most of the star formation within our sample is not highly obscured. This result is consistent with that of Prescott et al. (2007), who find that highly obscured star-forming regions make up only $\sim 3 \%$ of a sample of $\sim 1800$ star-forming regions within SINGS galaxies on scales down to $\sim 500 \mathrm{pc}$.

Comparing the parameters of radius and $K_{s}$ surface brightness, we find that the residual dispersions are similar. This result is not too surprising as the $K_{s}$ surface brightness samples the older stellar population of a galaxy that, generally, has a smooth spatial distribution as a function of radius. We also find that the residual dispersion around the trend of $q_{70}$ versus $I_{\nu}(24 \mu \mathrm{m}) / I_{\nu}(70 \mu \mathrm{m})$ is only slightly smaller than the dispersion in $q_{70}$ and significantly larger than the residual dispersion around the trend of $q_{70}$ versus $I_{\nu}(24 \mu \mathrm{m})$, suggesting that $q_{70}$ is not very sensitive to temperature changes.

\subsection{Local FIR/Radio Ratio and Star Formation}

The above results demonstrate that, at least on kiloparsec scales, the local FIR-radio correlation is strongly coupled to star formation activity. However, from an empirical standpoint, identifying physical parameters to minimize the residual dispersion in $q_{70}$ is not where the greatest improvement has been found. While we find an average decrease in the residual dispersion between $q_{70}$ and $24 \mu \mathrm{m}$ surface brightness of $\sim 20 \%$, M06b has shown that a phenomenological smearing model improves the correlation between the FIR and radio emission distributions of galaxies by a factor of $\sim 1.8$. This significantly larger improvement using the image-smearing technique likely arises because this method more precisely characterizes the time evolution of a galaxy's FIR and radio emission distributions, which depend critically on its star formation history. The image-smearing procedure
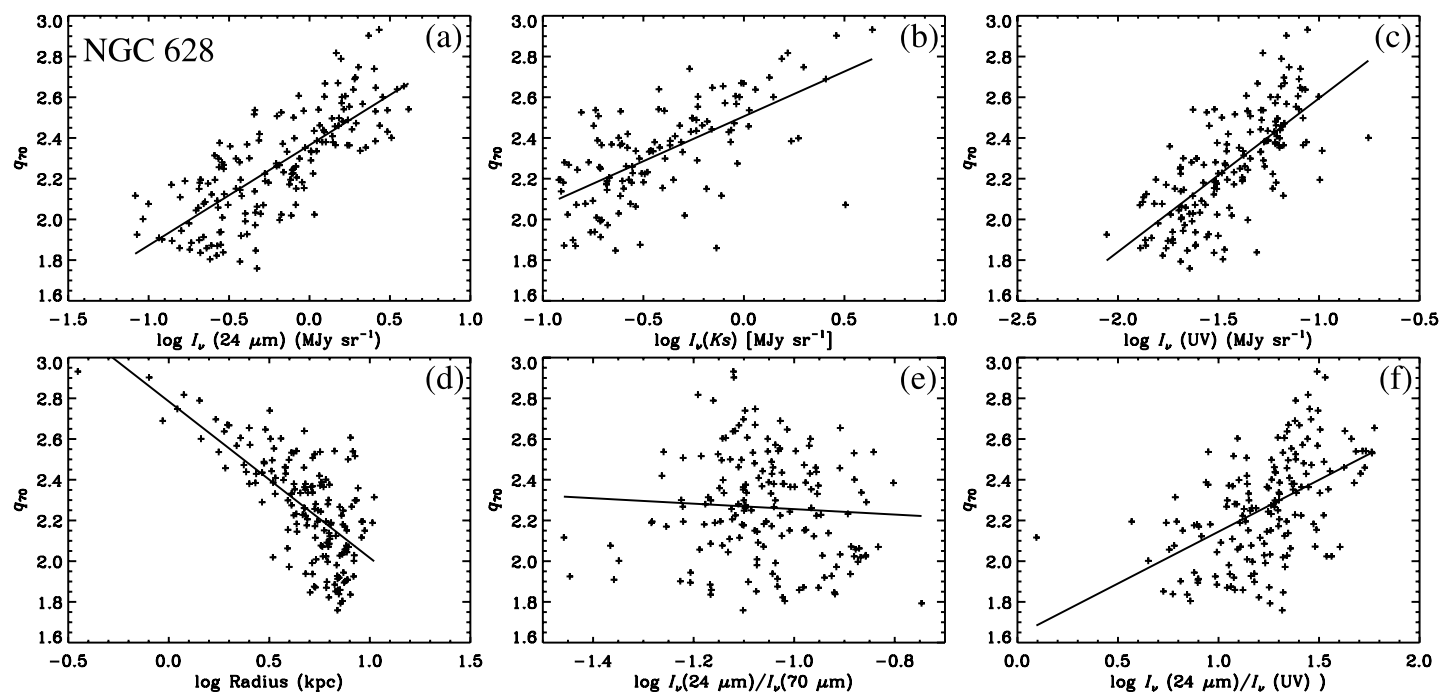

FIG. 2.-Plots comparing the dependence of $q_{70}$ on (a) $24 \mu \mathrm{m}$ surface brightness, (b) $K_{s}$ surface brightness, (c) GALEX UV surface brightness (FUV+NUV), (d) galactocentric radius, $(e)\left[I_{\nu}(24 \mu \mathrm{m}) / I_{\nu}(70 \mu \mathrm{m})\right]$ flux density ratio, and $(f)\left[I_{\nu}(24 \mu \mathrm{m}) / I_{\nu}(\mathrm{UV})\right]$ flux density ratio for NGC 628 . Each data point corresponds to a measurement from $1 \mathrm{kpc}^{2}$ pixels. OLS fits, which were used to measure the residual dispersion, are overplotted in each panel. 
TABLE 4

Residual Dispersion Results

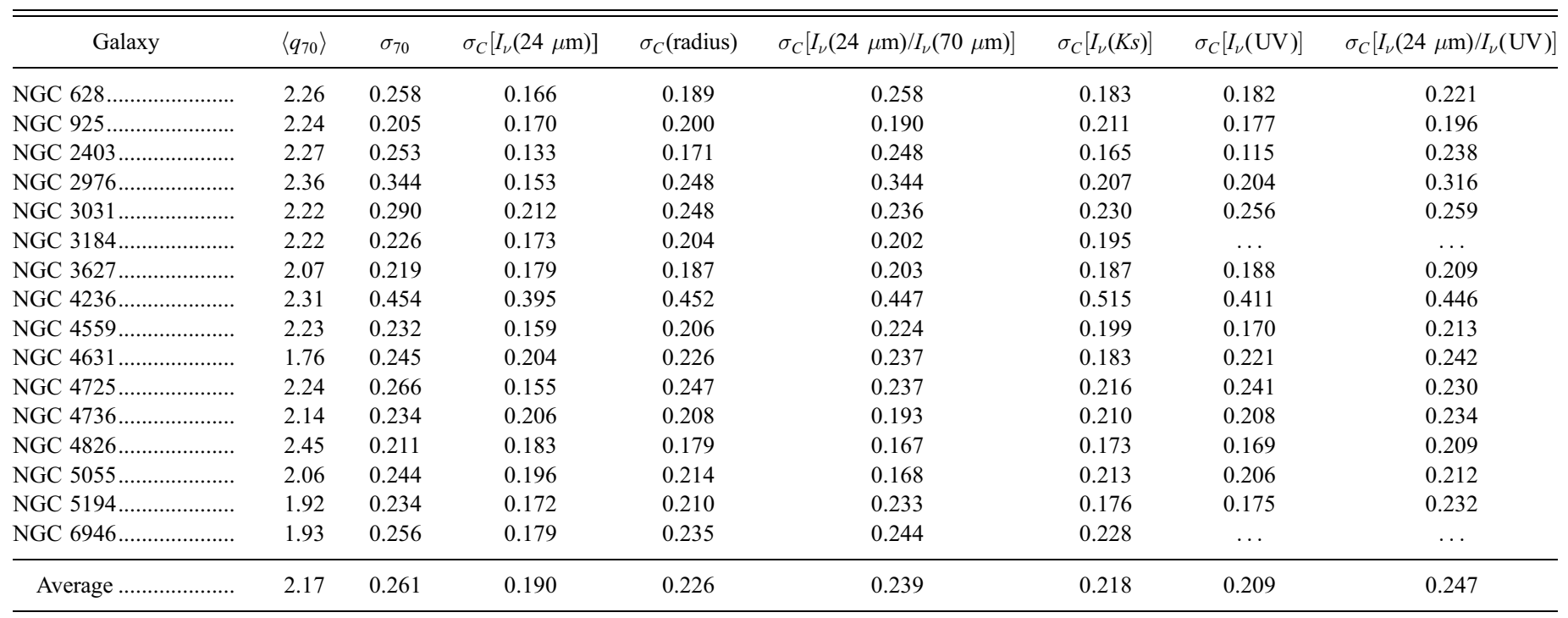

minimizes the residuals between a galaxy's infrared and radio images after smoothing the infrared map by a parameterized kernel assumed to contain the diffusion and decay characteristics of a galaxy's CR electrons. Since CR electrons spread over time, the size of each smoothing kernel is a measure of diffusion and decay that captures the time dependence mentioned above. Other parameters (e.g., density, magnetic field strength) can affect the permeability of the ISM to CR electrons and impede their propagation; such parameters will vary with $\mathrm{CR}$ electron location, from the vicinity of star-forming complexes to the more diffuse regions of the ISM. As the image-smearing picture appears to provide the best quantitative description for the FIR-radio correlation, we now introduce a more sophisticated approach to take into account the differences among $\mathrm{CR}$ electron populations within galaxy disks.

\section{TWO-COMPONENT IMAGE-SMEARING ANALYSIS}

Using a phenomenological image-smearing model, first presented by Bicay \& Helou (1990), M06b studied how the spatial distributions of FIR and radio emission varied as a function of star formation intensity within 12 spiral galaxy disks. Their results were then related to the physical model of Helou \& Bicay (1993). Although this method was found to improve the spatial correlation between the distributions of infrared and radio emission by reducing the residuals in the ratio maps, a single kernel failed to work perfectly for an entire galaxy disk as evidenced by the recognizable structures (i.e., spiral arms) left in the residual maps. This is not too surprising as the freshly injected CR electron populations associated with star-forming regions should be different in mean age and energy than those populations associated with a galaxy's diffuse disk; such CR electrons have likely lost a significant amount of their initial energy and/or have undergone reacceleration by passing interstellar shocks (e.g., Strong \& Moskalenko 1998; Berezhko et al. 2003). Consequently, it appears that a more realistic description should require a multiscale analysis of the galaxy images allowing for the separation of structures at various spatial frequencies. We now present such an analysis using a wavelet-based image decomposition technique and compare these results to those using a single-component method.

The simplest way to formulate a phenomenological model with two distinct CR electron populations is to create representa- tive source functions and allow them to be characterized by different scale lengths. We therefore decompose the infrared images (used as source proxies) into two components: one representing sites of ongoing star formation and the other consisting of diffuse emission from dust in the disk heated by older stars or from afar by young stars. While a purely spatial definition will not yield a perfect census of massive star formation sites, it has the advantage of simple assumptions and data manipulation.

\subsection{Choice of Separation Scale}

The goal of the decomposition is to separate each $70 \mu \mathrm{m}$ image into two components: one containing the smallest to largest physical structures associated with massive star formation and another to include a galaxy's diffuse FIR emission distribution. The typical distance for which $>90 \%$ of Lyman continuum photons are expected to be absorbed by interstellar gas and dust is $\sim 500$ pc (Dove \& Shull 1994); this value is in excellent agreement with the observed mean distances of diffuse ionized gas surrounding $\mathrm{H}$ in regions (Ferguson et al. 1996). A value of $1 \mathrm{kpc}$ is also a moderate upper limit to the sizes of giant $\mathrm{H}$ II regions and molecular cloud complexes, as well as widths of spiral arms. Furthermore, the results presented by M06b hinted that the bestfit global scale lengths seemed to be spread around a median value of $\sim 1 \mathrm{kpc}$ but did not cluster tightly around it. For these reasons we choose a disk-structure separation scale of $1 \mathrm{kpc}$. While this scale may not be perfect for all galaxies, it is physically motivated and allows for a uniform comparison among the nearest 18 WSRT-SINGS sample galaxies.

\subsection{Wavelet-based Image Decomposition}

The wavelet transform provides the ability to describe structures within an image as a function of their characteristic spatial scale. This property of the wavelet transform has led to the development of the wavelet-based Multiscale Vision Model (MVM; Bijaoui \& Rué 1995; Rué \& Bijaoui 1997), a procedure useful for identifying morphological features in astronomical images (e.g., Starck et al. 2000; Adami et al. 2005). Wavelet analyses have also been used to study the FIR-radio correlation within individual systems such as NGC 6946 (Frick et al. 2001), the LMC (Hughes et al. 2006), and M33 (Tabatabaei et al. 2007); in each of these studies a cross-correlation analysis was performed 

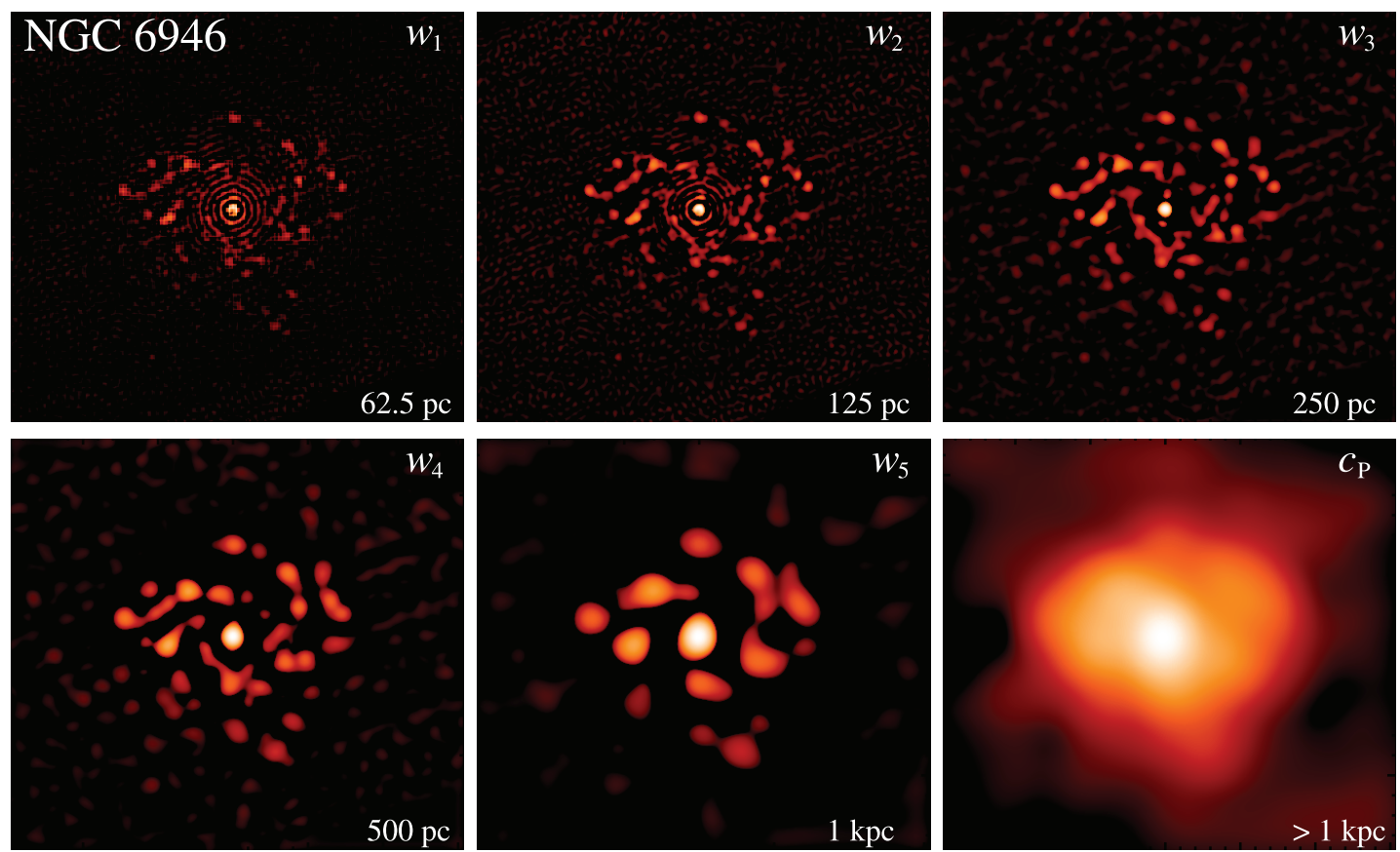

FIG. 3.-Wavelet transform of NGC 6946 at $70 \mu \mathrm{m}$ using the à trous algorithm (see $\S 5.2$ ). In this instance the image was regridded such that each wavelet plane $w_{p=1-5}$ corresponds to $2^{p-1} \times 62.5 \mathrm{pc}$ and the final smoothed plane, $c_{P}$, contains information for the remaining larger spatial scales. For the actual creation of the structure and disk images used in the present analysis, the regridding scheme described in $\S 5.2$ was used, which ensures that the structure image is sampled at the Nyquist frequency. Each plane is displayed using a logarithmic stretch ranging from the $1 \sigma \mathrm{rms}$ level of the background to the maximum value of the wavelet coefficients at that plane. The observed $70 \mu \mathrm{m}$ image is recovered exactly by summing these six planes.

on the wavelet power spectra for images acquired at various wavelengths.

For the purpose of this study, we need only make use of the wavelet transform to separate structures in each image as a function of their characteristic spatial frequency. We apply the wavelet transform to our images using the à trous algorithm (Holdschneider et al. 1989; Bijaoui 1991). The à trous algorithm produces a set of wavelet coefficients $w_{p}$, at each scale $p$, having the same number of pixels as the original image, $c_{o}$. The exact physical scale corresponding to each wavelet plane, as indexed by $p$, will depend on the distance to the object and the pixel scale of the image. It then follows that a pixel $j$ of the observed image can be expressed as the sum of all the wavelet coefficients at this position, plus a final smooth plane containing the remaining largescale features, $c_{P}$, such that

$$
c_{o, j}=c_{P, j}+\sum_{p=1}^{P} w_{p, j} .
$$

An example of the wavelet transform via the à trous algorithm is given in Figure 3 for NGC 6946. The complete à trous algorithm, as well as detailed discussions on the applicability of wavelet transforms to astronomical data sets, can be found in Starck \& Murtagh (2002).

We decompose each observed infrared image, $I$, into two subimages: (1) a structure image containing features with spatial scale smaller than $1 \mathrm{kpc}$, and (2) a disk image containing all structures with characteristic spatial scales larger than or equal to $1 \mathrm{kpc}$, largely constituting a galaxy's diffuse disk. The à trous algorithm is dyadic, transforming the image at discrete scales that are powers of 2 and indexed here by $p$. We therefore regrid each image appropriately such that $2^{p-1}$ pixels, where $p \geq 3$, corresponds to $1 \mathrm{kpc}$ at a given galaxy's distance. This regridding scheme ensures that the structure image is sampled at the
Nyquist frequency and provides uniformity in the decompositions for all galaxies. The final structure and disk images, $I_{\text {str }}$ and $I_{\mathrm{dsk}}$, respectively, are then created by summing the appropriate scales such that

$$
I_{\mathrm{str}}=\sum_{p=1}^{p(<1 \mathrm{kpc})} w_{p}
$$

and

$$
I_{\mathrm{dsk}}=I_{P}+\sum_{p(\geq 1 \mathrm{kpc})}^{P} w_{p},
$$

where $I_{P}$ is the final smooth plane containing all remaining largescale features and $I=I_{\mathrm{str}}+I_{\mathrm{dsk}}$. For illustration, we display in Figure 4 the structure and disk images at $70 \mu \mathrm{m}$ for three galaxies (NGC 628, NGC 5055, and NGC 2976) chosen to span a large fraction of our sample's range in infrared surface brightness. Each panel is displayed with the same stretch to allow comparison between the morphology and amount of power residing in the disk and structure components as a function of infrared surface brightness.

\subsection{Correcting for Free-Free Emission}

The image-smearing analysis is designed to compare the spatial distributions of a galaxy's FIR and nonthermal radio emission. Because the observed radiation at $22 \mathrm{~cm}$ is a combination of nonthermal (synchrotron) and thermal (free-free) emission, we estimate and subtract the thermal fraction of radio emission from the observed $22 \mathrm{~cm}$ maps. This is done by estimating the free-free emission using a scaled version of $24 \mu \mathrm{m}$ maps as described in M06b. 

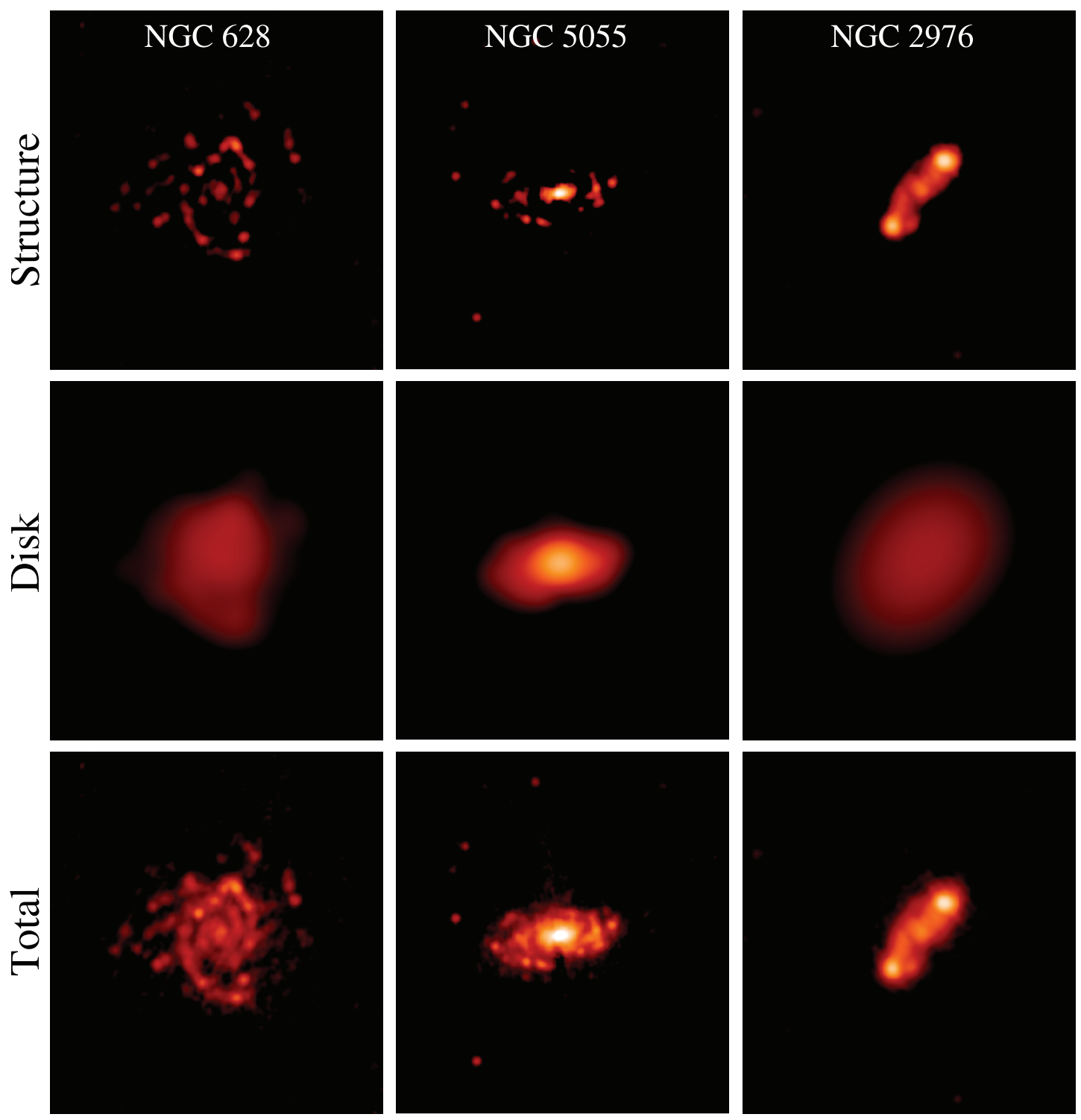

FIG. 4.-Structure, disk, and total (observed) $70 \mu \mathrm{m}$ images of NGC 628, NGC 5055, and NGC 2976. These galaxies were chosen to span our sample's range in infrared surface brightness and are ordered, left to right, by increasing radiation field energy density. We have used a common logarithmic scaling for all six panels running from the $3 \sigma$ level in the noisiest map to the maximum surface brightness level among all the maps. The structure image contains all features significant at spatial frequencies less than $1 \mathrm{kpc}$, while the disk image contains all features significant at spatial scales greater than or equal to $1 \mathrm{kpc}$. (See $\S 5.2 \mathrm{for}$ details.)

The scaling factor relies on the empirical correlation found to exist between $24 \mu \mathrm{m}$ and extinction-corrected $\mathrm{Pa} \alpha$ luminosities within NGC 5194 (M51a) by Calzetti et al. (2005). While it has been demonstrated that this correlation is not universal (e.g., Pérez-González et al. 2006; Calzetti et al. 2007), it has proven to be more than sufficient as a first-order estimate of the thermal radio emission (M06b). Having estimated the $\mathrm{Pa} \alpha$ line emission from the observed $24 \mu \mathrm{m}$ emission, we can then determine the corresponding ionizing photon rate (Osterbrock 1989) and expected free-free emission at $1.4 \mathrm{GHz}$ (Rubin 1968) such that

$$
\left(\frac{S_{T}}{\mathrm{Jy}}\right) \sim 7.93 \times 10^{-3}\left(\frac{T}{10^{4} \mathrm{~K}}\right)^{0.45}\left(\frac{\nu}{\mathrm{GHz}}\right)^{-0.1}\left[\frac{f_{\nu}(24 \mu \mathrm{m})}{\mathrm{Jy}}\right],
$$

where we have assumed an average $\mathrm{H}$ II region temperature of $T=10^{4} \mathrm{~K}$.
To check the reliability of this method for estimating the radio thermal fractions, we compare our results to those of Niklas et al. (1997) for 12 galaxies that appear in both samples. The thermal fractions of Niklas et al. (1997) were derived by the completely independent method of radio spectral index fitting. We list the 1.0 GHz thermal fractions of Niklas et al. (1997) along with our estimated thermal fractions, after scaling our $1.4 \mathrm{GHz}$ flux densities to what is expected at $1.0 \mathrm{GHz}$ assuming a mean spectral index of -0.8 for the nonthermal component, in Table 5 . We find that, except for the cases of NGC 4736 and NGC 5033, our estimated thermal fractions are very similar to those reported by Niklas et al. (1997). We note that NGC 5033 hosts a Seyfert 2 type nucleus, which could complicate this method of free-free estimation using the $24 \mu \mathrm{m}$ imaging.

\subsection{Phenomenological Image-smearing Model}

The basic procedure used here is similar to that presented in M06a and M06b. We calculate the residuals between the free-free 
TABLE 5

Radio Thermal Fraction Comparison

\begin{tabular}{|c|c|c|}
\hline \multirow[b]{2}{*}{ GaLAXY } & \multicolumn{2}{|c|}{$f_{T}^{1 \mathrm{GHz}}$} \\
\hline & Niklas et al. (1997) & This Paper \\
\hline 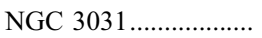 & 0.05 & 0.06 \\
\hline 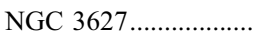 & 0.09 & 0.09 \\
\hline 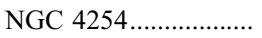 & 0.05 & 0.05 \\
\hline 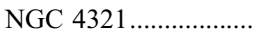 & 0.05 & 0.06 \\
\hline 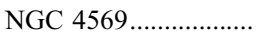 & 0.07 & 0.07 \\
\hline 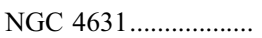 & 0.03 & 0.04 \\
\hline 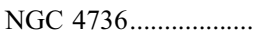 & 0.18 & 0.10 \\
\hline 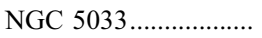 & 0.11 & 0.05 \\
\hline NGC $5055 \ldots \ldots \ldots \ldots \ldots . . . . . . . .$. & 0.09 & 0.08 \\
\hline 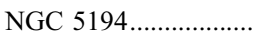 & 0.05 & 0.06 \\
\hline 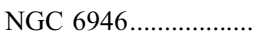 & 0.06 & 0.07 \\
\hline 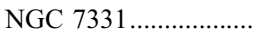 & 0.06 & 0.06 \\
\hline
\end{tabular}

corrected radio and observed infrared images after convolving the infrared maps by a parameterized kernel $\kappa(\boldsymbol{r})$. The new element is that we now smear $I_{\text {str }}$ and $I_{\text {dsk }}$ independently, then add the two smeared images and compare the sum to the radio image. The smoothing kernel is a function of a two-dimensional position vector $\boldsymbol{r}$, having a magnitude $r=\left(x^{2}+y^{2}\right)^{1 / 2}$, where $x$ and $y$ are the right ascension and declination offsets on the sky, respectively.

Due to the large range in inclination among our sample galaxies, we compare results for exponential smoothing kernels oriented either in the plane of the galaxy disk or isotropically. An exponential kernel was chosen because it was found to work as well as, or better than, Gaussian kernels by M06a. This is consistent with "leaky box" type models where CR electron escape is expected to occur on timescales less than or comparable to the diffusion timescales (Bicay \& Helou 1990). The kernel takes the form $\kappa(\boldsymbol{r})=e^{-\boldsymbol{r} / \boldsymbol{r}_{0}}$, where $\boldsymbol{r}_{0}$ is its $e$-folding length $l$ modified by geometric factors related to the position angle $\theta$ and inclination $i$ of a galaxy such that

$$
\boldsymbol{r}_{0}=\frac{l \cos i}{\left[1-(\boldsymbol{x} \sin \theta+\boldsymbol{y} \cos \theta)^{2} \sin ^{2} i / r^{2}\right]^{1 / 2}} .
$$

The position angle $\theta$ of the tilt axis of the galactic disk is measured east of north, and $i=0$ defines a face-on projection. Kernels are isotropic when $r_{0}=l$. This situation is the same as if a galaxy's inclination and position angle were equal to zero.

Let $R(\boldsymbol{r})$ denote the observed radio image and $I(\boldsymbol{r})=I_{\text {str }}(\boldsymbol{r})+$ $I_{\mathrm{dsk}}(\boldsymbol{r})$ denote the observed infrared image, which has been separated into component images containing structures larger or smaller than $\sim 1 \mathrm{kpc}$ (see $\S 5.2$ ). The two-dimensional residual function, defined by $\phi$, is calculated between the radio and smeared infrared images after the infrared disk and structure images are first smoothed by independent kernels and then summed. This residual surface is given by

$$
\phi\left(Q, l_{\mathrm{str}}, l_{\mathrm{dsk}}\right)=\frac{\sum\left[Q^{-1} \tilde{I}_{j}\left(l_{\mathrm{str}}, l_{\mathrm{dsk}}\right)-R_{j}\right]^{2}}{\sum R_{j}^{2}},
$$

where $Q=\sum I_{j}(\boldsymbol{r}) / \sum R_{j}(\boldsymbol{r})$ is used as a normalization factor [i.e., $\log Q=q_{\lambda}$ (global)],

$$
\tilde{I}\left(l_{\mathrm{str}}, l_{\mathrm{dsk}}\right)=\tilde{I}_{\mathrm{str}}\left(l_{\mathrm{str}}\right)+\tilde{I}_{\mathrm{dsk}}\left(l_{\mathrm{dsk}}\right)
$$

represents the infrared image after smearing $I_{\text {str }}$ and $I_{\text {dsk }}$ with independent kernels having scale length $l_{\text {str }}$ and $l_{\text {dsk }}$, respectively, and the subscript $j$ indexes each pixel. Our calculation of the residuals is slightly different than what has been used in M06a and M06b; we now fix the normalization factor, $Q$, rather than let it vary with the kernel size. This modification is necessary since we are now using different smoothing kernels for each component. The quantity $\phi$ was minimized to determine the best-fit smearing kernel for the structure and disk images of each galaxy in our sample. The normalization by the squared sum of the radio flux density allows for proper comparison of our galaxies, which vary in intrinsic surface brightness. We plot $\log \phi$ as a function of $l_{\mathrm{str}}$ and $l_{\mathrm{dsk}}$ in Figure 5. The special case of a single kernel, as studied by M06a and M06b, is reproduced by the diagonal cut of the residual surface plotted in Figure 5 (i.e., when $\left.l_{\text {str }}=l_{\text {dsk }}\right)$. These residual curves are presented in Figure 6 and binned by the amount of star formation activity within each galaxy.

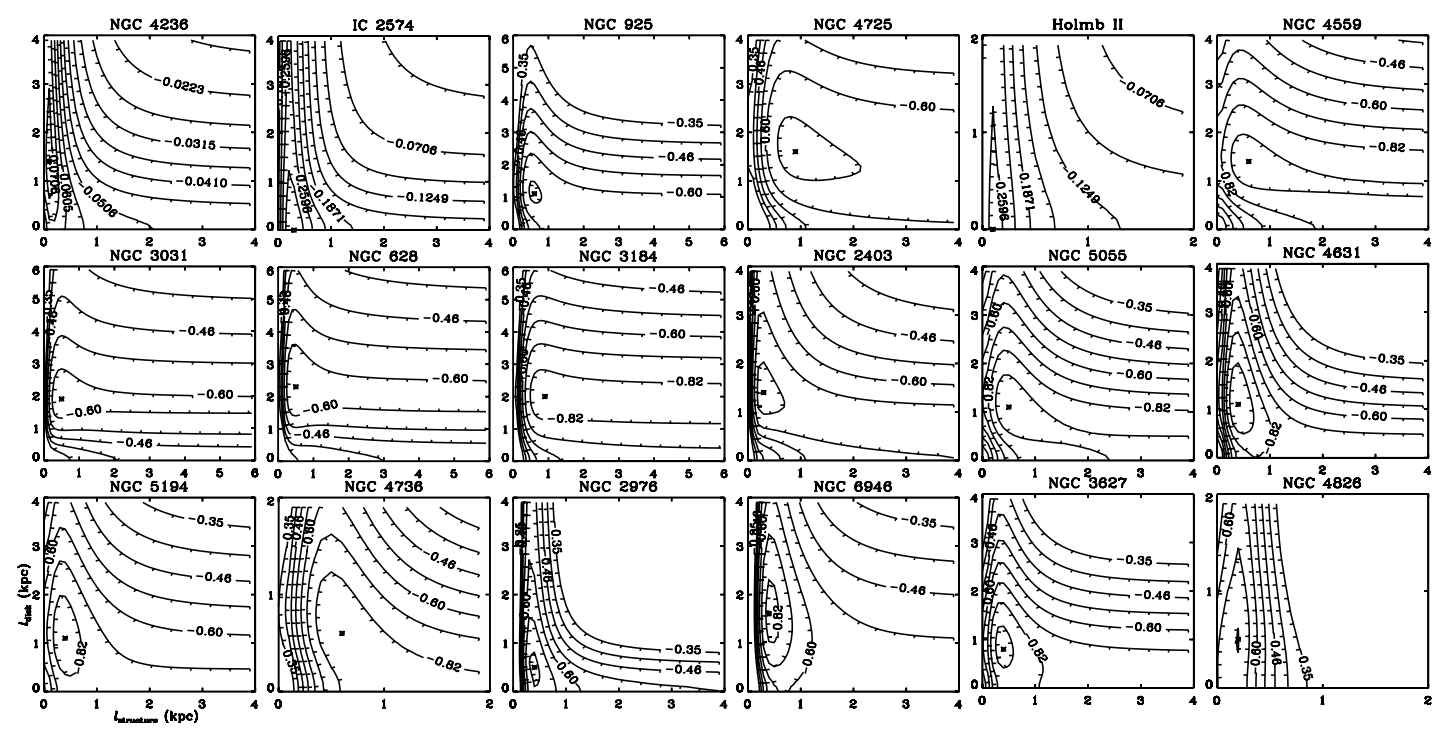

FIg. 5.-Residual surfaces for each galaxy, ordered by increasing $U_{\text {rad }}$ from left to right, top to bottom. The abscissa and ordinate designate the scale length of the kernel used to smooth the structure and disk component images, respectively. The minimum value on the surface is identified with an asterisk. A diagonal cut (i.e., where $\left.l_{\text {str }}=l_{\text {dsk }}\right)$ through each surface plot corresponds to the one-dimensional residual curves that are plotted in Fig. 6. 


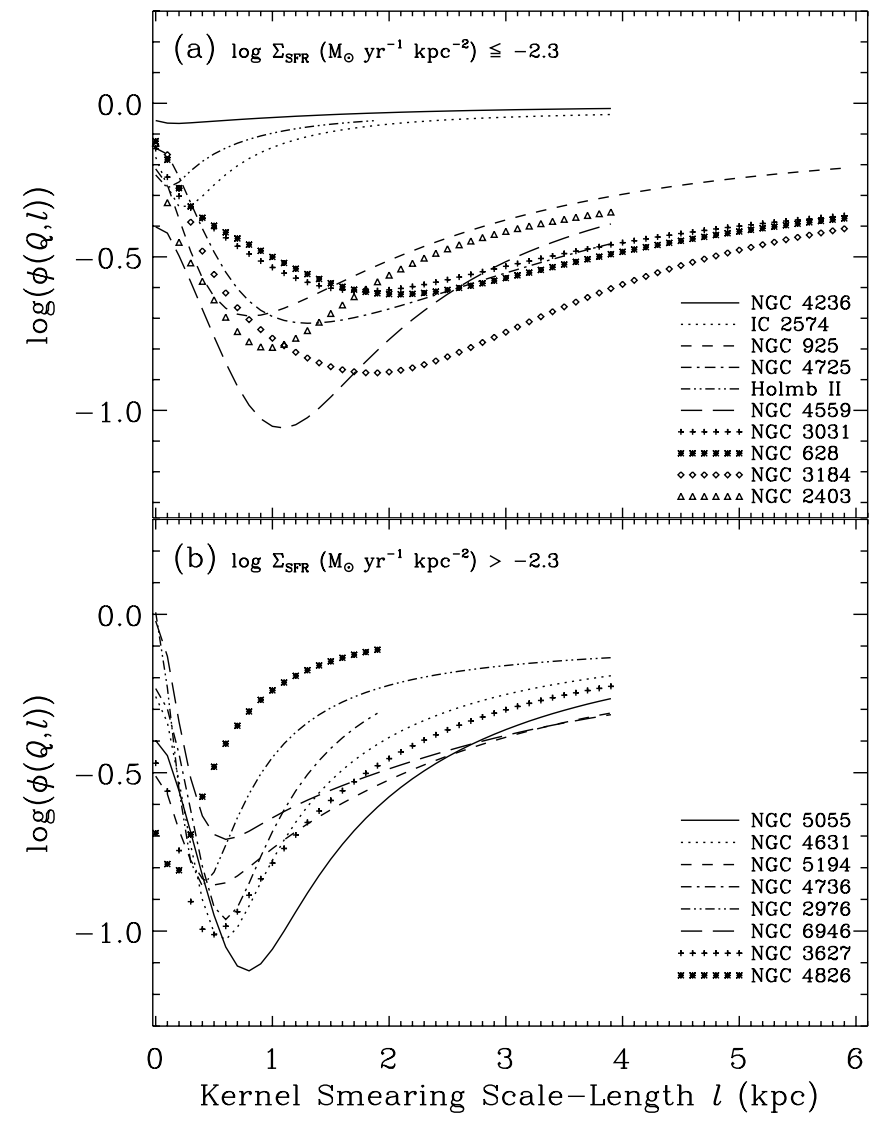

FIG. 6.- Residuals between the radio maps and smeared $70 \mu \mathrm{m}$ images (as defined in $\S 5.4$ ) as a function of smearing scale length. The results plotted here use isotropic kernels and $22 \mathrm{~cm}$ maps that have been corrected for the presence of free-free emission (see $\S 5.3$ ). (a) Galaxies having low disk-averaged SFRs, defined by $\log U_{\text {rad }} \leq-12.5=\log \Sigma_{\mathrm{SFR}} \leq-2.3$ (see $\left.\S 3.6\right) ;(b)$ galaxies with high star formation activity and larger values of $U_{\text {rad }}\left(\Sigma_{\mathrm{SFR}}\right)$.

The estimation of the residuals is carried out after first removing pixels that are not detected at the $3 \sigma$ level in either the radio or maximally smeared infrared maps and editing out contaminating background radio sources. Additional editing was necessary for NGC 3031, NGC 3627, NGC 4725, NGC 4736, and NGC 4826. Each of these galaxies hosts an active galactic nucleus (AGN). Because the phenomenology we are trying to test is strictly associated with the physics of star formation processes, we try to remove any contamination an AGN might introduce. This is done by first subtracting a scaled PSF from the nuclear region of the $70 \mu \mathrm{m}$ images before smoothing to remove any excess emission associated with the AGN. We then excise a $1 \mathrm{kpc}$ region around the nucleus before calculating the residuals between the smeared infrared and radio images. Since the AGNs within SINGS galaxies are all very low power, a $1 \mathrm{kpc}$ region should be adequate to remove the influence of an AGN. Additional editing was also necessary for NGC 5194; its companion galaxy (NGC 5195) was removed before calculating the residuals.

We use the quantity

$$
\Phi=\log \left\{\frac{\phi(Q, 0,0)}{\min \left[\phi\left(Q, l_{\mathrm{str}}, l_{\mathrm{dsk}}\right)\right]}\right\}
$$

which is the depth of the minimum value in the residual surface, as a measure of how much the correlation is improved by smooth- ing the infrared structure and disk images. In the special case when $l_{\text {str }}=l_{\mathrm{dsk}}$, we have

$$
\Phi_{\text {glob }}=\log \left\{\frac{\phi(Q, 0)}{\min \left[\phi\left(Q, l_{\mathrm{str}}=l_{\mathrm{dsk}}\right)\right]}\right\},
$$

which is the same $\Phi$ studied by M06a and M06b (i.e., the minima found for the diagonal residual curves presented in Fig. 6). We denote the best-fit scale length that maximizes $\Phi_{\text {glob }}$ as $l_{\text {glob }}$ and refer to this quantity as the best-fit global scale length for the galaxy. Uncertainties in $\phi$ are estimated by numerically propagating the uncertainties in the input $70 \mu \mathrm{m}$ and $22 \mathrm{~cm}$ images as measured by the $1 \sigma$ rms noise of each map; the uncertainty on the best-fit scale length is then estimated as the range in scale length corresponding to the range from $\min (\phi)$ to $\min (\phi)+$ unc $(\phi)$, spanning along the diagonal and structure and disk axes of the residual surface maps.

While $\phi$ and $\Phi$ characterize the residual behavior as single quantities, we also constructed residual images for each galaxy using the best-fit smearing kernels to inspect the spatial variations of the residuals using this new, two-component method. The residual images, defined by

$$
\text { Residual image }=\log \left[Q^{-1} \tilde{I}(\boldsymbol{r})\right]-\log [R(\boldsymbol{r})],
$$

are plotted in the fifth column of Figure 1 for isotropic kernels having an exponential profile as this kernel type was associated with the largest values of $\Phi$ (see $\S 6.3$ ). For a comparison, the residual maps associated with the best-fit global scale lengths are given in the fourth column of Figure 1.

An additional free parameter of the relative amplitude of the disk and structure components was considered for a subset of the sample spanning the full range in surface brightness. This was done to test whether the proposed two-component analysis alone is sufficient at capturing the signatures of CR electrons associated with a galaxy's structures and disk or whether the relative efficiencies between structure and disk processes in producing synchrotron emission are significant. This could be especially important in the cases for which the power in the structure and disk components is comparable. While slight quantitative improvements (i.e., on the few percent level) are seen, they were not dramatic enough to warrant extra free parameters.

\section{TWO-COMPONENT MODELING RESULTS}

While we have shown that the FIR/radio ratios measured within galaxies are most sensitive to star formation activity in $\S 4$, we note that the largest quantitative improvement to the dispersion in the FIR/radio ratio within galaxies is achieved via our image-smearing analysis (i.e., nearly a factor of 2 compared to a factor of $\sim 1.2$ using a residual dispersion analysis); this procedure describes the spreading and decay of CR electrons in galaxy disks. In fact, by repeating the correlation analysis described in $\S 4$ with the best-fit smoothed infrared maps, we find that the gradients seen between $q_{70}$ and $24 \mu \mathrm{m}$ surface brightness and radius are flattened, on average, by factors of 5 and 4 , respectively. Hence, our phenomenological model clearly captures the behavior observed in $\S 4$; infrared emission is more strongly peaked around star-forming regions since CR electrons diffuse away from bright structures into diffuse regions of the ISM.

In the following section we present the results from our twocomponent image-smearing analysis and look for the cases in which significant gains are obtained over the single-component 


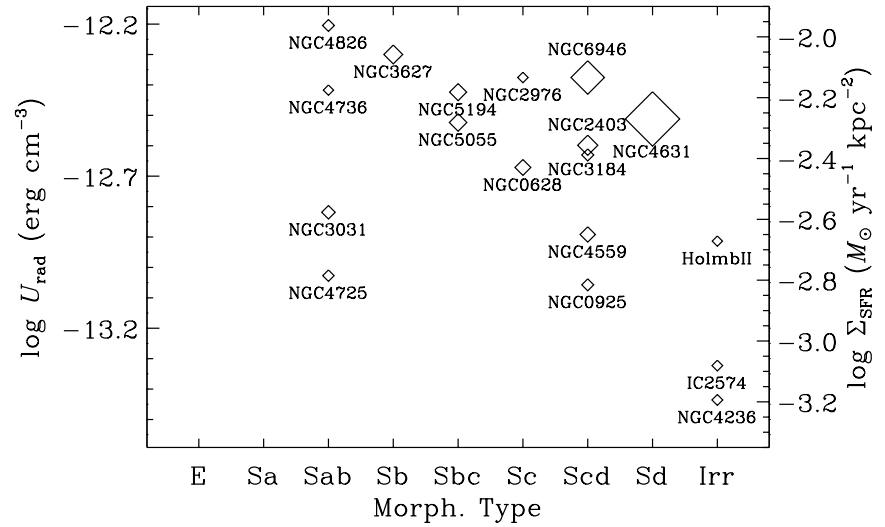

FIG. 7.- Radiation field energy density binned by morphological type. The plotting symbol sizes are scaled as a function of the improvement found using the two-component image-smearing procedure compared to using the singlecomponent method (i.e., $\Phi-\Phi_{\text {glob }}$ ). This value ranges from 0.00 dex for NGC 4736 to 0.24 dex for NGC 4631 .

method. Since this phenomenology is valid for nonthermal radio continuum emission, we focus our discussion on the results using radio images that have been corrected for the presence of free-free emission as described in $\S 5.3$ and note that this correction only slightly increases (i.e., $0.1 \mathrm{kpc}$ on average) the measured best-fit scale lengths.

\subsection{Comparison with Single-Component Method}

We find a range in the behavior of the residual surface maps among the sample galaxies in Figure 5. For nearly all galaxies we observe an initially steep (negative) gradient for increasing structure scale lengths as the minimum in $\log \phi$ is reached. Even though some galaxies may not exhibit a well-defined minimum along the structure axis (e.g., NGC 628), the steep initial gradient clearly demonstrates that the structure component is being detected by our two-component analysis. Similarly, many galaxies show a well-defined minimum along the disk axis (e.g., NGC 4559) while others do not (e.g., NGC 4631), suggesting that this component is evident as well. This diverse behavior among the residual maps demonstrates that our two-component analysis does yield qualitative insight into the two major CR electron populations under discussion.

To assess quantitatively the gains of using the two-component approach over image smearing with a single kernel, we look at the differences between $\Phi$ and $\Phi_{\text {glob }}$, which we give in Table 7 below. We find a large range in improvement spanning from 0.00 (NGC 4736) to 0.24 dex (NGC 4631). While galaxies showing the largest improvements generally have high infrared surface brightnesses, galaxies exhibiting very little improvement are well distributed as a function of infrared surface brightness. We also assess whether improvement correlates with morphology.

In Figure 7 we plot each galaxy's radiation field energy density as a function of morphological type; the plotting symbol sizes are proportional to $\Phi-\Phi_{\text {glob }}$. Irregulars and early-type spirals show essentially no improvement by using the two-component approach. Significant improvement appears limited to spiral galaxies of type $\mathrm{Sb}$ and later with high surface brightnesses.

The negligible improvement found for irregulars $\left(\Phi-\Phi_{\text {glob }} \lesssim\right.$ 0.005 dex) can be explained by their lack of a diffuse emission component at both FIR and radio wavelengths. This is clearly seen by inspecting the $70 \mu \mathrm{m}$ and $22 \mathrm{~cm}$ images of these galaxies in Figure 1. As a consequence of this, these galaxies have been excluded in the statistical comparisons of best-fit scale lengths presented in $\S \S 6.3$ and 6.4; these galaxies will be revisited in $\S 7.3$.
As for the early-type spirals, small $\left(\Phi-\Phi_{\text {glob }} \lesssim 0.02 \mathrm{dex}\right) \mathrm{im}-$ provements are probably expected since their FIR and radio emission is dominated by an inner disk; if the central concentration of emission largely dominates over that of the disk, this morphology will be pretty well described by a single component compared to a galaxy disk containing many organized star-forming structures. Consequently, we find that the two-component approach has mainly improved our phenomenological description of the FIR-radio correlation for late-type galaxies exhibiting clear spiral structure and significant star formation throughout their disks; this corresponds to roughly half the sample spirals, for which $\left\langle\Phi-\Phi_{\text {glob }}\right\rangle \sim 0.1$ dex.

A discrepancy is found for NGC 2976, which is a member of the M81 group. This discrepancy may be due to the fact that the galaxy's optical morphology (which is classified as an Sc peculiar) is strikingly different than its morphology in the FIR. In the FIR its disk emission arises from a very small disk $\left(D_{\mathrm{TIR}} \sim\right.$ $6 \mathrm{kpc}$ ) and is dominated by two large star-forming complexes on either side of a relatively weak nucleus; these features are almost unnoticeable in the optical. In fact, detailed studies of NGC 2976 carried out in the optical (Bronkalla et al. 1992) and in H I (Stil \& Israel 2002) have shown that the galaxy has dwarflike characteristics and possibly triggered star formation due to encounters between other members in the central M81 group. Furthermore, the optical luminosity, and presumably mass, of NGC 2976 is much smaller than that for the sample spirals, but similar to the sample irregulars. Such findings are consistent with our result; NGC 2976 behaves more like the irregulars in the sample for which we find little improvement is obtained by using the twocomponent analysis compared to the single-component method.

\subsection{Morphological Differences in Residual Images}

The residual images using the single-component and twocomponent analyses are displayed in the fourth and fifth columns of Figure 1, respectively. As already stated in $\S 6.1$, we find that the decrease in the residuals between the FIR and radio maps for the two-component analysis with respect to the single-component analysis is significantly greater for late-type spirals having a relatively large amount of ongoing star formation.

The residual images using the single-component method display a general trend such that galaxies with low star formation activity exhibit radio excesses associated with star-forming regions while galaxies with higher star formation activity behave in an opposite manner; these galaxies display infrared excesses associated with star-forming regions in their residual images. This is the result of galaxies with low star formation activity generally needing large scale lengths to best match their infrared and radio spatial distributions, leading to oversmoothed star-forming regions relative to the more actively star-forming galaxies. Now, by using the two-component technique and smoothing small spatial scale features (i.e., individual star-forming complexes) with smoothing functions independent of those used for the large spatial frequency components, this trend has been largely suppressed for a number of galaxies (i.e., NGC 4631, NGC 5194, NGC 6946).

A comparison of the residual images for our sample's nearest grand-design spiral (NGC 6946) in Figure 1 best illustrates this result; the rather dramatic differences in the residual behavior for star-forming spiral arms and quiescent interarm regions, which are evident when using a single kernel, are largely suppressed by our new treatment. Some organized structure still remains in our two-component residual image, which is not surprising since this method only looks to characterize the extremes of a galaxy's CR electron population. The remaining structures might also suggest 


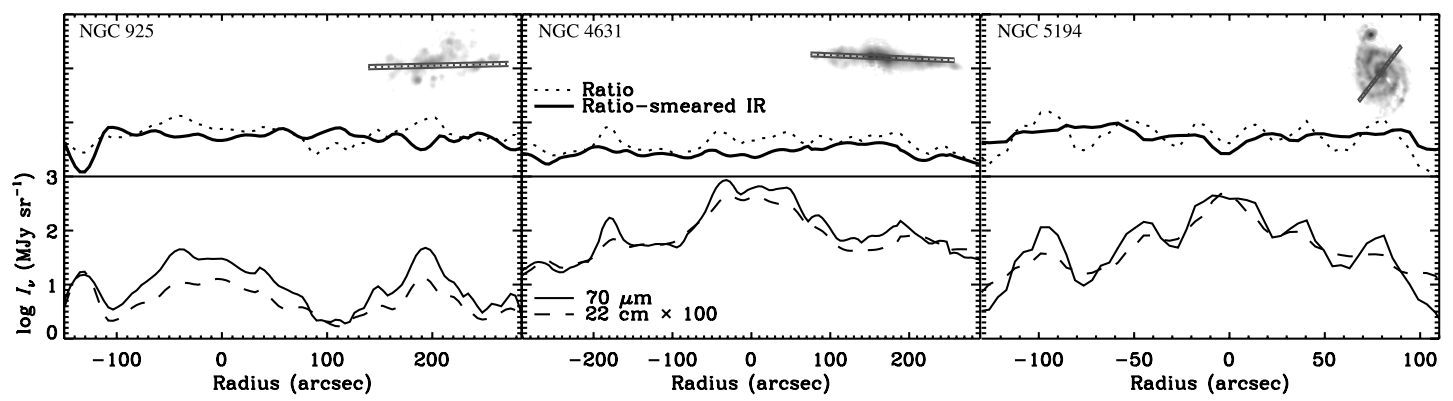

FIG. 8. - In the bottom portion of each panel we plot radial cuts across the $70 \mu \mathrm{m}$ and $22 \mathrm{~cm}$ maps of NGC 925 , NGC 4631 , and NGC 5194 . The $22 \mathrm{~cm}$ profile has been scaled by a factor of 100 to allow for a better comparison against the $70 \mu \mathrm{m}$ profile. In the top portion of each panel we plot the ratio between the radio and the observed infrared profiles along with the ratio between the radio the infrared profiles after smoothing by the best-fit kernels. Each cut, displayed in the thumbnail image of the galaxy, has a width equal to the FWHM of the $70 \mu \mathrm{m}$ PSF. [See the electronic edition of the Journal for a color version of this figure.]

that our symmetric smoothing functions have limited applicability; diffusion preferentially occurring along field lines, which trace arms and become tangled in star-forming regions, will lead to asymmetries associated with spiral arm structure (e.g., NGC 6946; Beck 2007).

Since it is difficult to determine amplitude information from the residual images in Figure 1, we show radial cuts across the $70 \mu \mathrm{m}$ and $22 \mathrm{~cm}$ maps, along with the ratios between the radio and both the observed and smoothed $70 \mu \mathrm{m}$ images, in Figure 8. This is done for three galaxies (NGC 925, NGC 4631, and NGC 5194) chosen to span our sample's morphology and inclination. These plots clearly illustrate that the amplitude of the variations in the FIR/radio ratios across each galaxy disk is significantly reduced by our image-smearing procedure. In the case of the grand-design spiral NGC 5194, we also find that the arms are broader in the radio compared to the FIR; this observation is consistent with CR electrons being accelerated in star formation sites within spiral arms and diffusing larger distances than associated dust heating photons.

\subsection{Inclination Effects}

We now determine how inclination affects the choice of smoothing kernel. In Table 6 we give the median values of $\Phi$ and $\Phi_{\text {glob }}$ for isotropic or in-disk kernels after separating the sample into low- $\left(i \leq 60^{\circ}\right)$ and high-inclination $\left(i>60^{\circ}\right)$ bins.

As expected, the difference for face-on galaxies appears almost negligible; the median $\delta \Phi=\operatorname{med}\left(\Phi_{\text {iso }}-\Phi_{\text {gal }}\right)$ for these galaxies is $\sim 0.02 \mathrm{dex}$, while $\delta \Phi_{\text {glob }}$ is also $\sim 0.02$ dex. Conversely, if a galaxy's inclination is greater than $\sim 60^{\circ}$, then the orientation of the kernel seems to become important. In this case, isotropic kernels are strongly favored such that $\delta \Phi$ is $\sim 0.17 \mathrm{dex}$ while $\delta \Phi_{\text {glob }}$ is $\sim 0.13$ dex.

The regular component of a galaxy's magnetic field is spread most densely throughout its thin disk; the radial diffusion of CR electrons should then preferentially occur along field lines, while vertical (out-of-plane) diffusion should require CR electrons to undergo an increased amount of cross field diffusion. Such a scenario has been verified empirically. Indirect estimates of diffusion coefficients for the vertical propagation of CR electrons in a galaxy's thin disk have been found to be an order of magnitude smaller than those for radial diffusion (Dahlem et al. 1995).

However, in galaxies with active star formation, ordered magnetic fields can be ruptured, allowing CR electrons to quickly escape their disks and form synchrotron halos (e.g., Hummel et al. 1988). This decrease in vertical confinement will lead to a diffusion behavior that is more isotropic in appearance. A clear example of this scenario is seen in the nearly edge-on galaxy, NGC 4631. NGC 4631 has a large radio halo that extends $\sim 5 \mathrm{kpc}$ beyond the vertical extent of the FIR disk (Dahlem et al. 1995). Our results therefore suggest that the highly inclined sample galaxies each possess, at least to some degree, synchrotron halos in which the diffusion of CR electrons occurs on similar timescales as those in the disk.

\subsection{Best-Fit Scale Lengths}

In Figure 5 we plot the residual surfaces associated with the two-component image-smearing procedure as described in $\S 5.4$. The best-fit disk, structure, and global scale lengths, along with estimated errors (see $\S 5.4$ ), are given in Table 7 . We find that the best-fit scale lengths for the structure images are on average $\sim 0.5 \mathrm{kpc}$ while the best-fit scale lengths for the disk images are, on average, $\sim 1.3 \mathrm{kpc}$; or, in other words, the best-fit disk scale length is, on average, $\sim 2.5$ times larger than the best-fit structure scale length. The best-fit global scale lengths, using the singlecomponent approach, are found to be $\sim 1.0 \mathrm{kpc}$, on average.

To ensure that the exact separation scale used in decomposition of the $70 \mu \mathrm{m}$ images does not affect the best-fit disk and structure scale lengths, we repeated our analysis using separation scales ranging from 0.5 (for galaxies resolved at this scale) to $2.0 \mathrm{kpc}$ for four galaxies of varying size, morphology, and infrared surface brightness (i.e., NGC 628, NGC 2976, NGC 2403, NGC 6946). We find that the dispersion in best-fit disk and structure scale lengths is $\sim 0.15$ and $0.10 \mathrm{kpc}$, respectively. Our separation method therefore seems quite robust, and the best-fit disk and structure scale lengths appear to be fairly insensitive to the exact separation scale.

TABLE 6

Effects of Inclination on Kernel Shape for Sample Spirals

\begin{tabular}{|c|c|c|c|c|c|c|c|}
\hline \multirow{2}{*}{$\begin{array}{l}\text { INCLINATION } \\
\quad(\mathrm{deg})\end{array}$} & \multirow[b]{2}{*}{$N$} & \multicolumn{2}{|c|}{ Median $\Phi($ dex $)$} & \multirow[b]{2}{*}{$\delta \Phi$} & \multicolumn{2}{|c|}{ Median $\Phi_{\text {glob }}($ dex $)$} & \multirow[b]{2}{*}{$\delta \Phi_{\text {glob }}$} \\
\hline & & Iso & Gal & & Iso & Gal & \\
\hline$\leq 60$ & 11 & 0.59 & 0.57 & 0.02 & 0.57 & 0.55 & 0.02 \\
\hline$>60$ & 4 & 0.87 & 0.61 & 0.17 & 0.77 & 0.56 & 0.13 \\
\hline
\end{tabular}


TABLE 7

Two-Component Fitting Results

\begin{tabular}{|c|c|c|c|c|c|c|}
\hline Galaxy & $\begin{array}{l}l_{\text {glob }} \\
(\mathrm{kpc})\end{array}$ & $\begin{array}{c}l_{\text {str }} \\
(\mathrm{kpc})\end{array}$ & $\begin{array}{c}l_{\mathrm{dsk}} \\
(\mathrm{kpc})\end{array}$ & $\begin{array}{l}\Phi_{\text {glob }} \\
(\text { dex })\end{array}$ & $\begin{array}{c}\Phi \\
(\mathrm{dex})\end{array}$ & $\begin{array}{c}\Phi-\Phi_{\text {glob }} \\
\quad(\text { dex })\end{array}$ \\
\hline 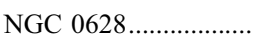 & $2.1_{-0.61}^{+0.73}$ & $0.5_{-0.18}^{+0.28}$ & $2.3_{-0.52}^{+0.60}$ & 0.499 & 0.561 & 0.063 \\
\hline 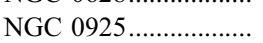 & $0.8_{-0.21}^{+0.33}$ & $0.6_{-0.20}^{+0.37}$ & $1.1_{-0.42}^{-0.52}$ & 0.479 & 0.501 & 0.022 \\
\hline 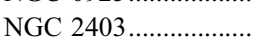 & $1.0_{-021}^{+0.216}$ & $0.3_{-0.10}^{+0.16}$ & $1.4_{-0.32}^{-0.32}$ & 0.664 & 0.762 & 0.098 \\
\hline 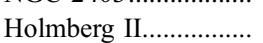 & $0.1_{-0.04}^{+0.21}$ & $0.1_{-0.04}^{+0.10}$ & $0.0_{-\mathrm{NaN}}^{+1.32}$ & 0.043 & 0.043 & 0.000 \\
\hline 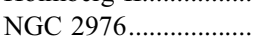 & $0.4_{-0.01}^{-0.04}$ & $0.4_{-0.03}^{+0.04}$ & $0.5_{-0.20}^{+0.16}$ & 0.864 & 0.867 & 0.003 \\
\hline 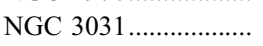 & $1.8_{-0.58}^{+0.73}$ & $0.5_{-0.22}^{+0.59}$ & $1.9_{-0.44}^{-0.60}$ & 0.464 & 0.503 & 0.039 \\
\hline 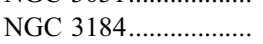 & $1.9_{-045}^{+0.39}$ & $0.9_{-0.66}^{+0.22}$ & $2.0_{-0.35}^{+0.44}$ & 0.741 & 0.764 & 0.023 \\
\hline IC 2574 & $0.2^{-0.17}$ & $0.3^{-0.11}$ & $0.0^{-0.60}$ & 0.161 & 0.167 & 0.006 \\
\hline 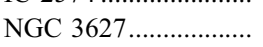 & $0.5_{-0.01}^{+0.03}$ & $0.4_{-0.03}^{+0.013}$ & $0.8_{-0.07}^{-0.13}$ & 0.541 & 0.629 & 0.088 \\
\hline 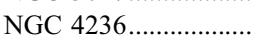 & $0.2_{-0.16}^{-0.010}$ & $0.1_{-0.12}^{+0.12}$ & $1.4_{-1.02}^{+3.01}$ & 0.009 & 0.017 & 0.008 \\
\hline 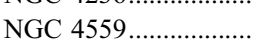 & $\begin{array}{l}1 .-0.16 \\
1.1_{-0.05}^{+0.03}\end{array}$ & $0.6_{-0.11}^{+0.12}$ & $1.4_{-0.14}^{-0.02}$ & 0.655 & 0.713 & 0.058 \\
\hline 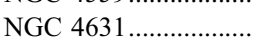 & $0.6_{-0.09}^{+0.055}$ & $0.4_{-0.00}^{+0.01}$ & $1.1_{-0.06}^{-0.05}$ & 0.768 & 1.009 & 0.241 \\
\hline 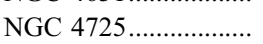 & $1.3_{-047}^{+0.84}$ & $0.9_{-041}^{+3.94}$ & $1.6_{-078}^{+0.98}$ & 0.570 & 0.585 & 0.015 \\
\hline 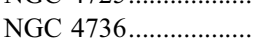 & $0.6_{-0.01}^{+0.02}$ & $0.6^{-0.03}$ & $0.6_{-0.07}^{+0.11}$ & 0.729 & 0.729 & 0.000 \\
\hline 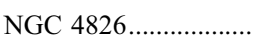 & $0.2_{-0.04}^{-0.01}$ & $0.2_{-0.04}^{-0.04}$ & $0.5_{-025}^{-0.023}$ & 0.117 & 0.138 & 0.021 \\
\hline NGC 5055 & $0.8_{-0.09}^{+0.06}$ & $0.5_{-0.00}^{+0.01}$ & $1.1_{-0.03}^{+0.02}$ & 0.727 & 0.805 & 0.078 \\
\hline NGC 5194_............ & $0.5_{-0.04}^{+0.10}$ & $0.4_{-0.05}^{+0.01}$ & $1.1_{-014}^{+0.13}$ & 0.343 & 0.417 & 0.073 \\
\hline 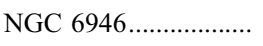 & $0.6_{-0.10}^{+0.14}$ & $0.4_{-0.00}^{+0.02}$ & $1.6_{-0.11}^{+0.10}$ & 0.690 & 0.860 & 0.171 \\
\hline Average $^{\mathrm{a}}$. & 0.9 & 0.5 & 1.3 & 0.590 & 0.656 & 0.066 \\
\hline
\end{tabular}

${ }^{\text {a }}$ The irregular galaxies (Holmberg II, IC 2574, and NGC 4236) were excluded when calculating the averages.

In Figure $9 a$ we plot the best-fit disk and structure scale lengths versus radiation field energy density, which we also express as a star formation surface density. Also included in this plot are the best-fit scale lengths found using the single-component method. We perform ordinary least-squares fits to the best-fit disk, structure, and global scale lengths with $U_{\text {rad }}$ as the independent variable. The least-squares fits for the best-fit disk and structure scale lengths in Figure $9 a$ have very similar slopes $(-0.43$ and -0.47 , respectively). Furthermore, the best-fit global scale lengths appear to be nearer to the best-fit disk scale lengths for galaxies having low values of $U_{\text {rad }}$ and nearer to the best-fit structure scale lengths for galaxies having high values of $U_{\text {rad }}$. This result is consistent with another observation, namely, that the best-fit global scale lengths display a general trend of decreasing with increasing ratio of the power in the $70 \mu \mathrm{m}$ structure to disk components. These results suggest that the difference between the FIR and radio emission spatial distributions is dependent on whether the disk or structure is the dominant emission component of a galaxy. In $\S 7$ we propose that the disk will generally be composed of a population of CR electrons that are significantly older than those that have been recently injected into the ISM and still reside close to their parent star-forming regions.

We also note that four galaxies (NGC 925, NGC 4725, NGC 4559 , and NGC 3031) appear to be off of the general trend between
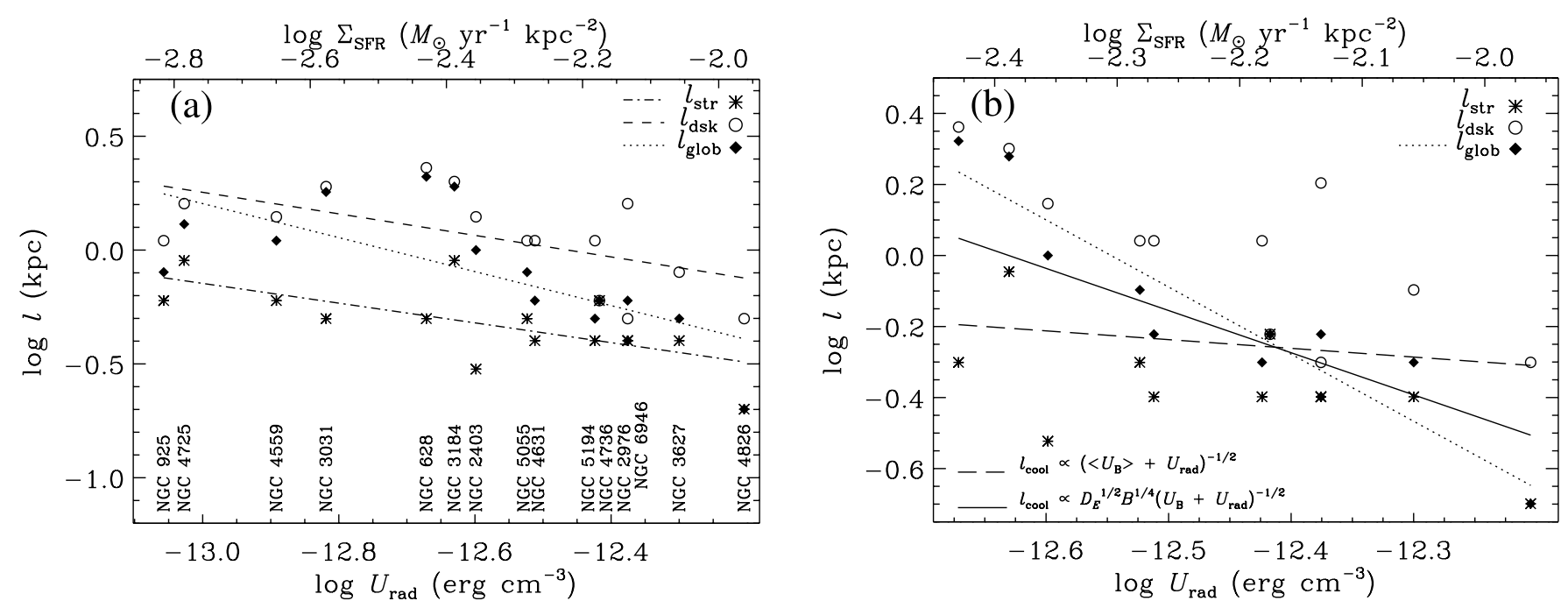

FIG. 9.- In panel (a) we plot the best-fit disk, structure, and global scale lengths for galaxies in our sample that are resolved at scales less than 1 kpc against the

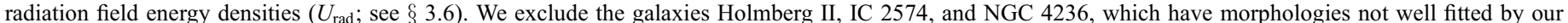

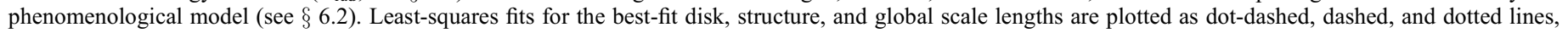

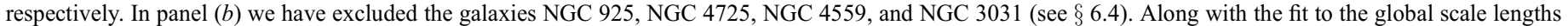

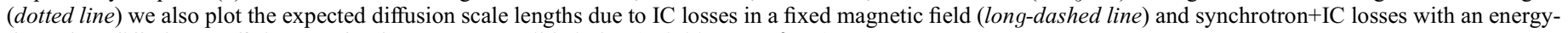
dependent diffusion coefficient $D_{E}$ for the steepest possible index (solid line; see $\S 7.1$ ). 
best-fit global scale length and star formation rate surface density first identified by M06b. While the best-fit structure scale lengths of these galaxies appear consistent with the rest of the sample, the best-fit disk and global scale lengths appear to be lower than what one would expect compared to the trend for the higher surface brightness galaxies. Of the 15 galaxies we plot in Figure $9 a$, these four galaxies also have the four lowest surface brightnesses. This turnover at $\log U_{\text {rad }} \sim-12.7$ may be due to a signal-tonoise ratio effect; specifically, we may not be detecting the most diffuse emission in the extended parts of these galaxy disks, which is throwing off our estimations for the best-fit scale lengths. For example, by truncating the areas over which we calculate the residuals, the turnover is shifted toward values of $\log U_{\text {rad }}>$ -12.7. Conversely, simulations of much deeper observations to detect a very low surface brightness disk lead to longer scale lengths, suggesting again that the turnover may be due to the limited sensitivity of our data. On the other hand, the location of these galaxies in Figure $9 a$ may not be a signal-to-noise ratio artifact, but due to a different physical makeup of these systems. Because it is difficult to settle this issue without deeper observations, we include a discussion on the physical implications of this scenario in $\S 7.3$.

\section{DISCUSSION}

We find that the FIR and nonthermal radio morphologies are more similar to each other for galaxies having higher radiation field energy densities compared to galaxies with lower radiation field energy densities. Following the interpretation of M06b, our results indicate that $\mathrm{CR}$ electrons are, on average, closer to their place of origin in galaxies having higher star formation activity.

Since the diffusion scale length of CR electrons depends only on their age and ability to diffuse through the ISM of galaxies, there are four possible explanations of the results. The CR electrons within galaxies having high star formation activity may (1) have relatively short lifetimes due to a high energy loss rate; (2) diffuse at a slower rate due to the ISM having a high density and magnetic field strength, resulting in a shorter mean free path; (3) escape into intergalactic space at a higher rate due to an increased occurrence of ruptured magnetic field lines (this explanation implies systematically higher global FIR/radio ratios); or (4) have been accelerated recently and be relatively young. The first three of these explanations are applicable in the case of steady state star formation (i.e., the variation of ISM parameters alone will lead to shorter scale lengths). The fourth explanation, however, requires a recent episode of enhanced star formation to inject fresh CR electrons into a galaxy's ISM. We try to distinguish among these four scenarios in the context of the results for the two-component smearing analysis.

\subsection{CR Electron Cooling Timescales and Diffusion Properties in Normal Galaxies}

We now describe the physical processes associated with the propagation and decay of CR electrons in the ISM. We derive CR electron cooling timescales and introduce a diffusion equation to derive propagation distances and physical scaling relations for comparison with our observations. These comparisons will allow us to discriminate between the four scenarios above.

As CR electrons propagate through the ISM of galaxies, they lose their energy due to a number of physical processes, including synchrotron, inverse Compton (IC) scattering, bremsstrahlung, ionization, and adiabatic expansion losses. In normal galaxies synchrotron and IC scattering processes are likely the most significant energy loss terms for CR electrons associated with $1 \mathrm{GHz}$ emission (Condon 1992); the other terms listed will become nonnegligible, however, for galaxies hosting extreme episodes of star formation like starbursting ultraluminous infrared galaxies (ULIRGs; e.g., Thompson et al. 2006). We now derive the CR electron cooling timescales associated with the two dominant processes.

Let us assume that $\mathrm{CR}$ electrons propagating with a pitch angle $\alpha$ in a magnetic field of strength $B$ have isotropically distributed velocities such that $\left\langle\sin ^{2} \alpha\right\rangle=\frac{2}{3}$, leading to $B_{\perp} \approx 0.82 B$. According to synchrotron theory, a CR electron having energy $E$ will emit most of its energy at a critical frequency $\nu_{c}$, where

$$
\left(\frac{\nu_{c}}{\mathrm{GHz}}\right)=1.3 \times 10^{-2}\left(\frac{B}{\mu \mathrm{G}}\right)\left(\frac{E}{\mathrm{GeV}}\right)^{2} .
$$

Since the energy loss of CR electrons by synchrotron radiation goes as $d E / d t \propto U_{B} E^{2}$, we can use equation (17) to express the synchrotron cooling timescale, $\tau_{\text {syn }} \equiv E /|d E / d t|_{\text {syn }}$, for CR electrons as

$$
\begin{aligned}
\left(\frac{\tau_{\text {syn }}}{\mathrm{yr}}\right) \sim & 5.7 \times 10^{7}\left(\frac{\nu_{c}}{\mathrm{GHz}}\right)^{-1 / 2}\left(\frac{B}{\mu \mathrm{G}}\right)^{1 / 2} \\
& \times\left(\frac{U_{B}}{10^{-12} \mathrm{ergs} \mathrm{cm}^{-3}}\right)^{-1},
\end{aligned}
$$

where $U_{B}=B^{2} /(8 \pi)$ is the magnetic field energy density. Naturally, we can rewrite the synchrotron cooling timescale as

$$
\left(\frac{\tau_{\text {syn }}}{\mathrm{yr}}\right) \sim 1.4 \times 10^{9}\left(\frac{\nu_{c}}{\mathrm{GHz}}\right)^{-1 / 2}\left(\frac{B}{\mu \mathrm{G}}\right)^{-3 / 2} .
$$

Similarly, since the energy loss of CR electrons due to IC scattering goes as $d E / d t \propto U_{\text {rad }} E^{2}$, we can again use equation (17) to write the IC cooling timescale, $\tau_{\text {IC }} \equiv E /|d E / d t|_{\text {IC }}$, as

$$
\begin{aligned}
\left(\frac{\tau_{\mathrm{IC}}}{\mathrm{yr}}\right) \sim & 5.7 \times 10^{7}\left(\frac{\nu_{c}}{\mathrm{GHz}}\right)^{-1 / 2}\left(\frac{B}{\mu \mathrm{G}}\right)^{1 / 2} \\
& \times\left(\frac{U_{\mathrm{rad}}}{10^{-12} \mathrm{ergs} \mathrm{cm}^{-3}}\right)^{-1}
\end{aligned}
$$

where $U_{\text {rad }}$ is the radiation field energy density. Photons with a frequency $\nu_{p}$ will significantly contribute to IC losses if below the Klein-Nishina limit; for $\mathrm{GeV}$ electrons considered here, the upper photon energy limit lies in the X-ray regime (i.e., $h \nu_{p} \lesssim$ $200 \mathrm{eV}$ ), but the bulk of losses arise from interactions with IR/ optical photons, which dominate $U_{\text {rad }}$. The effective cooling timescale for CR electrons due to synchrotron and IC losses is

$$
\tau_{\text {cool }}^{-1}=\tau_{\text {syn }}^{-1}+\tau_{\text {IC }}^{-1}
$$

which, by combining equations (18) and (20), we can express as

$$
\begin{aligned}
\left(\frac{\tau_{\text {cool }}}{\mathrm{yr}}\right) \sim & 5.7 \times 10^{7}\left(\frac{\nu_{c}}{\mathrm{GHz}}\right)^{-1 / 2}\left(\frac{B}{\mu \mathrm{G}}\right)^{1 / 2} \\
& \times\left(\frac{U_{B}+U_{\mathrm{rad}}}{10^{-12} \mathrm{ergs} \mathrm{cm}^{-3}}\right)^{-1} .
\end{aligned}
$$

In simple diffusion models, the propagation of $\mathrm{CR}$ electrons is usually characterized by an empirical, energy-dependent diffusion coefficient, $D_{E}$ (e.g., Ginzburg et al. 1980). The value of $D_{E}$ 
has been found to be around (4-6) $\times 10^{28} \mathrm{~cm}^{2} \mathrm{~s}^{-1}$ for $\lesssim \mathrm{GeV}$ CRs by fitting diffusion models with direct measurements of $\mathrm{CR}$ nuclei (i.e., secondary-to-primary ratios like boron to carbon) within the solar neighborhood (e.g., Jones et al. 2001; Moskalenko et al. 2002; Maurin et al. 2002). While this empirically measured value is for CR nuclei within the Milky Way, it has been found to be consistent with inferred diffusion coefficients for CR electrons both radially $\left(\sim 10^{29} \mathrm{~cm}^{2} \mathrm{~s}^{-1}\right)$ and vertically $\left(\sim 10^{28} \mathrm{~cm}^{2} \mathrm{~s}^{-1}\right)$ in the thin disks of galaxies (i.e., NGC 891 and NGC 4631; Dahlem et al. 1995). We note that a value of $\sim 10^{29} \mathrm{~cm}^{2} \mathrm{~s}^{-1}$ is also found for hydrodynamic simulations of bubble/superbubble-induced galaxy outflows in studies of galaxy cluster abundances (e.g., Rebusco et al. 2005; Roediger et al. 2007). Since the mean modelderived diffusion coefficient, using direct measurement of $\mathrm{CR}$ nuclei, is similar to the mean value of inferred radial and vertical CR electron diffusion coefficients by Dahlem et al. (1995), we simply use

$$
\left(\frac{D_{E}}{\mathrm{~cm}^{2} \mathrm{~s}^{-1}}\right) \sim \begin{cases}5 \times 10^{28}, & E<1 \mathrm{GeV} \\ 5 \times 10^{28}\left(\frac{E}{\mathrm{GeV}}\right)^{1 / 2}, & E \geq 1 \mathrm{GeV} .\end{cases}
$$

Now, neglecting escape and using a simple random walk equation, we assume that $\mathrm{CR}$ electrons will diffuse a distance $l_{\text {cool }}=\left(D_{E} \tau_{\text {cool }}\right)^{1 / 2}$ before losing all of their energy to synchrotron and IC losses. By combining equations (17) and (23), we can express $D_{E}$ as a function of $B$ for a fixed $\nu_{c}$ such that, for CR electrons having energies $\geq 1 \mathrm{GeV}$,

$$
\left(\frac{l_{\text {cool }}}{\mathrm{kpc}}\right) \sim 7 \times 10^{-4}\left(\frac{\tau_{\text {cool }}}{\mathrm{yr}}\right)^{1 / 2}\left(\frac{\nu_{c}}{\mathrm{GHz}}\right)^{1 / 8}\left(\frac{B}{\mu \mathrm{G}}\right)^{-1 / 8} .
$$

\subsubsection{Order-of-Magnitude Estimates}

Using the above equations, we derive simple, order-of-magnitude estimates to determine whether diffusion and cooling of CR electrons in a steady state star formation model are able to account for our observations. In the following sections, more careful analyses are presented. Taking the mean value of $U_{\text {rad }}$ for those galaxies plotted in Figure $9 b$ (i.e., $3.7 \times 10^{-13} \mathrm{ergs} \mathrm{cm}^{-3}$ ) and assuming $U_{\text {rad }}=U_{B}$, which has been shown to be a reasonable assumption for a large sample of spiral galaxies (Lisenfeld et al. 1996b), we find from equation (22) that the average cooling time for a $1.4 \mathrm{GHz}-$-emitting $\mathrm{CR}$ electron is $\sim 1.1 \times 10^{8} \mathrm{yr}$. Inserting this value into equation (24), we measure a diffusion scale length of $\sim 6.8 \mathrm{kpc}$; this value is clearly off of the scale shown in Figure $9 b$. On the other hand, if we instead assume a fixed, typical magnetic field strength of $9 \mu \mathrm{G}$ (Niklas 1995) and that $U_{B}=U_{\mathrm{rad}}=3.2 \times 10^{-12} \mathrm{ergs} \mathrm{cm}^{-3}$, the average cooling time for a $1.4 \mathrm{GHz}$-emitting $\mathrm{CR}$ electron is $\sim 2.2 \times 10^{7} \mathrm{yr}$ with a diffusion scale length of $\sim 2.6 \mathrm{kpc}$. While this value for $U_{\mathrm{rad}}$ is much higher than what we infer from the average TIR surface brightness of the sample, it must apply near bright star-forming structures, whose TIR surface brightnesses are much greater. Even so, this diffusion scale length is much larger than any value we find for the best-fit structure scale lengths. From these simple order-of-magnitude estimates it appears that particle fading due to cooling by IC and synchrotron processes alone cannot explain the structural differences between the FIR and radio maps.

While CR electron escape may help to reconcile this discrepancy between the observed scale lengths and these order-ofmagnitude diffusion scale length calculations, we might then expect to find systematic variations in global FIR/radio ratios with best-fit scale lengths. No such trend is found, suggesting that escape is not a dominant variable among the galaxies included in Figure $9 b$. The role of escape, however, may be important for the sample irregulars, which is discussed in $\S 7.3$. For the sample spirals, the best explanation seems to be differences in the CR electron population ages.

\subsubsection{Comparison with Scaling Relations}

We now introduce a more detailed model to see if we can reproduce the observed trends in Figure $9 b$ by varying relevant ISM parameters using the above-mentioned scaling relations. Again setting $U_{B}=U_{\text {rad }}$ leads 5 to $B \propto U_{\text {rad }}^{1 / 2}$ and $\tau_{\text {cool }} \propto B^{-3 / 2}$. Since $D_{E} \propto l_{\mathrm{mfp}}$ and $l_{\mathrm{mfp}} \propto n^{\delta}$, where $l_{\mathrm{mfp}}$ is the mean free path traveled by CR electrons, $n$ is the ISM density, and $\delta$ depends on the specifics of the scattering processes within a system, we can estimate the relative importance of $B$ and $n$ on the distance CR electrons emitting at a characteristic frequency travel. We introduce the scaling relations

$$
B \propto n^{\beta}, \quad D_{E} \propto n^{\delta} B^{-1 / 4},
$$

where $\frac{1}{3} \leq \beta \leq \frac{2}{3}$ and $-1 \leq \delta \leq-\frac{1}{3}$ are realistic index ranges (Helou \& Bicay 1993), thereby leading to the dependence

$$
l_{\text {cool }} \propto U_{\text {rad }}^{1 / 4(\delta / \beta-7 / 4)} .
$$

While the range of $\beta$ has been derived empirically (e.g., Niklas $\&$ Beck 1997) and is consistent with the assumption of equipartition between turbulent kinetic energy of gas clouds and magnetic energy, as well as with dynamo models (e.g., Ruzmaikin et al. 1988), the values of $\delta$ are determined by the dominant CR electron scattering processes. A value of -1 describes how diffusion would occur for hard scattering off particles (i.e., the hard sphere approximation). On the other hand, $\delta=-\frac{1}{3}$ best describes soft scattering off of structures, specifically, the case in which scattering is dominated by localized $B$ perturbations whose spatial density is proportional to the density of the gas (Helou \& Bicay 1993). In reality, both scattering processes are likely active, which will correspond to an intermediate value of $\delta$; this value will likely vary within and among galaxies.

Excluding those galaxies that may suffer from low signal-tonoise ratio effects, we first take the simplest case and consider whether the observed trends in Figure $9 b$ may arise from an increase of IC losses alone within galaxies that are more actively forming stars. Fixing $B$, we plot the expected relation between $l_{\text {cool }}$ and $U_{\text {rad }}\left[\right.$ i.e., $l_{\text {cool }} \propto\left(\left\langle U_{B}\right\rangle+U_{\text {rad }}\right)^{-1 / 2}$ ] as a dashed line in Figure $9 b$. The fits to the global, structure, and disk data have slopes that are $\sim 7.7,2.7$, and 5.1 times steeper, respectively, than the slope of this line. It is probably more physical for each parameter, not just $U_{\text {rad }}$, to scale with the amount of star formation activity within a galaxy; we now investigate such a case.

Using the derived scaling relation given in equation (26) and taking $\beta=\frac{1}{3}$ and $\delta=-1$, we plot the expected trend between $l_{\text {cool }}$ and $U_{\text {rad }}$ in Figure $9 b$ as a solid line. These extreme choices of $\beta$ and $\delta$ correspond to the steepest possible slope and are unlikely to best describe most galaxies. To more easily compare this model with the fits to the disk, structure, and global scale length data, we plot the slopes of each fit in terms of $\delta$ and $\beta$ (as given in the exponent of eq. [26]) in Figure 10. The slope of a given line in

\footnotetext{
${ }^{5}$ We note that this relation between $B$ and $U_{\text {rad }}$ is slightly steeper than the $B \propto U_{\mathrm{rad}}^{1 / 3}$ scaling reported by Niklas \& Beck (1997). If we were to instead use this scaling, the final relation in eq. (26) would be less steep and even more discrepant with the fit to our observations in Fig. $9 b$.
} 


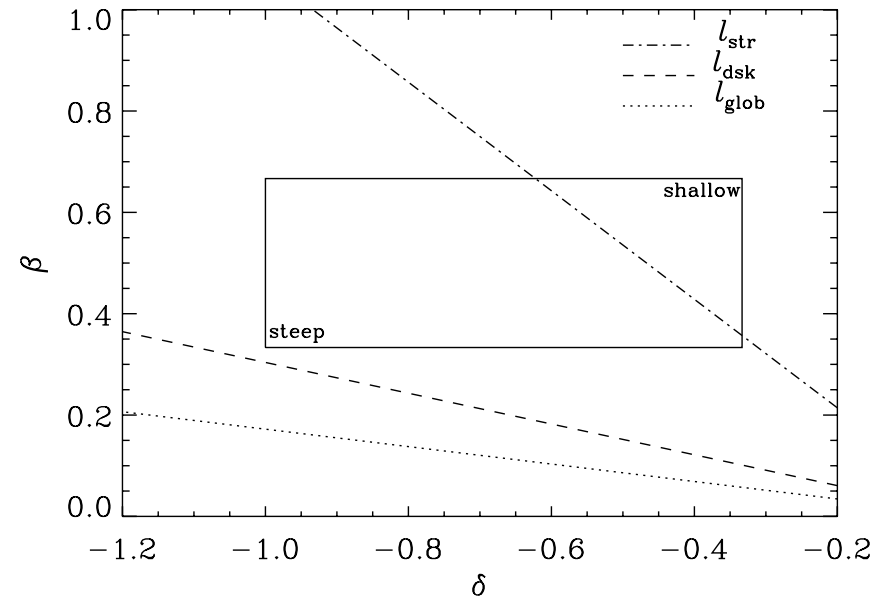

FIG. 10.- Slopes of the fits to global, structure, and disk scale lengths as a function of $U_{\text {rad }}$ (only for those galaxies shown in Fig. 9b), plotted in terms of $\delta / \beta$ as given in eq. (26). The overplotted box corresponds to the physically acceptable ranges of $\delta$ and $\beta$ (see $\S 7.1$ ).

Figure 10 corresponds to a line in Figure $9 b$ described by setting its slope equal to the exponent in equation (26). The physically acceptable ranges of $\delta$ and $\beta$ are given by the overplotted rectangle. The steepest and shallowest gradients correspond to coordinates of the lower left and upper right corners of the rectangle, respectively.

Only the fit to the structure scale length data passes through the region of physically acceptable values for $\beta$ and $\delta$. In particular, nearly the entire range of $\beta$-values and values of $\delta \gtrsim-\frac{2}{3}$ appear to be acceptable. This suggests that diffusion occurring in star-forming regions is best characterized as having a significant contribution from soft scattering off of perturbations in the magnetic field. This scenario seems reasonable as the field near starforming regions is known to be amplified and highly turbulent (Beck et al. 1996).

The fit to the disk scale lengths passes very near the plotted region of physically acceptable scaling values in Figure 10. Specifically, values of $\beta=\frac{1}{3}$ (weak magnetic field structure) and $\delta=-1$ (hard scattering) appear the most relevant.

Finally, we find no physically acceptable combination of $\delta$ and $\beta$ to reproduce the trend found between $l_{\text {glob }}$ and $U_{\text {rad }}$. This result, in addition to the order-of-magnitude estimate in $\S 7.1 .1$, suggests that systematic variations in ISM parameters alone cannot properly describe the mean distance traveled by a galaxy's CR electrons; rather, the age of the population must be the dominant parameter.

\subsection{Timescales of Star Formation Episodes}

Assuming that $\mathrm{CR}$ electron age is the dominant parameter that determines the measured best-fit scale lengths, we can infer ages for the star formation episodes responsible for injecting the associated CR electron population into the ISM. We derive these ages from equation (24), substituting $l_{\text {cool }}$ values with the best-fit scale lengths.

The mean equipartition strength of the total magnetic field for typical spiral galaxies is found to be $\sim 9 \mu \mathrm{G}$ (Niklas 1995). In strong spiral arms, such as those of NGC 5194, the total field strength is found to be significantly larger at a value of $\sim 30 \mu \mathrm{G}$ (Fletcher et al. 2004). The field strength in more quiescent regions of galaxies, like that near the Sun, has typical values of $\sim 6 \mu \mathrm{G}$ (e.g., Strong et al. 2000). Let us assume that each of these characteristic magnetic field strengths corresponds to the typical

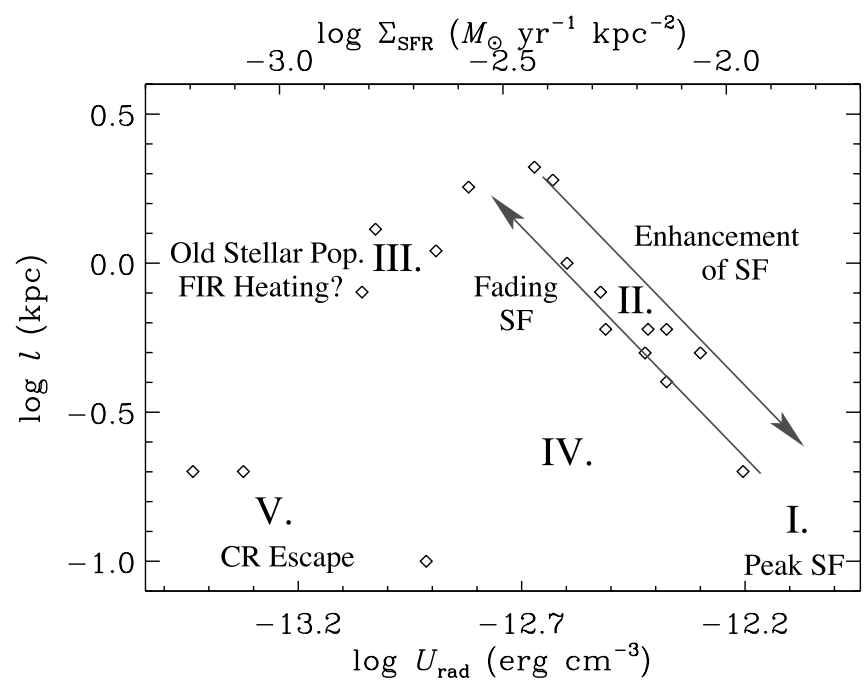

FIG. 11.-Best-fit global scale lengths for the entire 18 galaxy sample using isotropic kernels having an exponential profile and our free-free corrected radio maps. Overplotted on the scatter diagram are roman numerals to identify the general placement of galaxies on this diagram (see $\S 7.3$ for the full discussion). [See the electronic edition of the Journal for a color version of this figure.]

strengths for our global, structure, and disk components, respectively, and scales with $U_{\text {rad }}^{1 / 2}$ for the range in $U_{\text {rad }}$ found for the sample.

The estimated ages for the global CR electron populations among the sample galaxies are in the range $(0.14-14.) \times 10^{6} \mathrm{yr}$ with a mean age of $\sim 3.7 \mathrm{Myr}$. The ages for the CR electron populations associated with the disk and structure components are in the range $(0.77-15.) \times 10^{6} \mathrm{yr}$ and $(0.19-3.4) \times 10^{6} \mathrm{yr}$ and have mean values of $\sim 5.3$ and $1.2 \mathrm{Myr}$, respectively. Appropriately, we find the oldest ages to be larger than $10^{7} \mathrm{yr}$; this is approaching the average cooling time of a $1.4 \mathrm{GHz}$-emitting CR electron in a normal spiral galaxy. We also find that $\mathrm{CR}$ electrons residing in each galaxy's diffuse disk are $\gtrsim 4.4$ times older, on average, than the population of CR electrons associated with star-forming regions.

Furthermore, the galaxy exhibiting the highest star formation activity and shortest best-fit structure scale length appears to have a dominant $\mathrm{CR}$ electron population with an age of $\sim 2 \times$ $10^{5} \mathrm{yr}$. For this extreme case, such a young age suggests that the galaxy may still be in a phase of CR electron generation within the shells of SNRs; assuming an ambient particle density of $1 \mathrm{~cm}^{-3}$ and an explosion energy of $10^{51} \mathrm{ergs}$, this age is $\sim 5$ times larger than the adiabatic lifetime of SNRs, which by then should have expanded to have a diameter on the order of $\sim 60$ pc (e.g., Condon 1992).

\subsection{Morphologies of Star Formation and CR Escape}

In order to provide a physical interpretation for the location of galaxies in the best-fit scale length- $U_{\text {rad }}$ diagram, we describe their general locations, designated by roman numerals, in Figure 11. So far in this discussion, we have not considered the four lowest surface brightness galaxies (NGC 925, NGC 4725, NGC 4559, and NGC 3031) because their placement in Figure $9 a$ may be the result of a signal-to-noise ratio effect. Since it is difficult to determine whether this is in fact the case without having deeper imaging, we now speculate on a physical scenario that could explain their positions in Figure $9 a$.

Similarly, the irregular galaxies in our sample (Holmberg II, IC 2574, and NGC 4236) have also been excluded thus far in our 


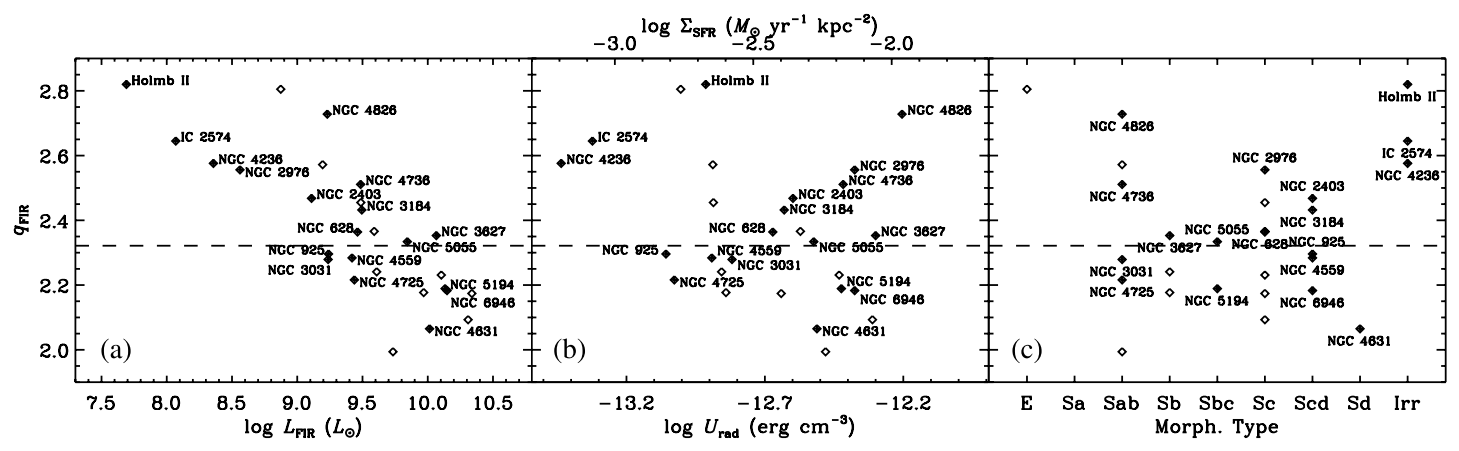

FIG. 12.-Global $q_{\text {FIR }}$ ratio for the entire WSRT-SINGS sample as a function of $(a)$ FIR luminosity, $(b)$ radiation field energy density, and $(c)$ morphological type. The horizontal line in each panel indicates the average $q_{\text {FIR }}$ ratio for the entire sample. Galaxies that are resolved at scales $\lesssim 1 \mathrm{kpc}$, and therefore included in the twocomponent image-smearing analysis, have been labeled.

discussion due to their lack of a disk component in the FIR and radio. The placement of these objects in Figure 11 and their behavior are now put into the context of our phenomenological picture:

1. Peak of most recent star formation event.-In this region of the diagram we assume that a galaxy has just reached a peak in its surface brightness after a recent episode of enhanced star formation; the episode must have occurred within the last few Myr to ensure that SNRs are still relatively young and that the morphologies of the radio and infrared emission are similar because CR electrons have not had time to diffuse very far. While the nucleus of NGC 6946 has been classified as a mild starburst (Ball et al. 1985), we note that our sample does not contain any systems for which the entire galaxy disk is experiencing a strong starburst. For systems experiencing such a mode of star formation, including the nuclear region of NGC 6946, high radiation fields and strong winds associated with the starburst could complicate this picture by rupturing magnetic field lines and increasing CR electron escape.

2. Enhancement and fading of star formation.-After reaching a peak in global star formation activity, those CR electrons associated with the recent enhancement of star formation will dominate the CR population. As they spread through the galaxy, they will begin to lose their energy to synchrotron and IC processes. In $\S 7.1$ we have demonstrated that a galaxy's position along this trend cannot be due to variations in ISM parameters alone; rather, its star formation history is the dominant parameter.

Alternatively, galaxies may move along this part of phase space as star formation activity becomes enhanced and then fades. Assuming a mean $B$ of $10 \mu \mathrm{G}$ and $U_{\text {rad }}=U_{B}, 1.4 \mathrm{GHz}$-emitting CR electrons should lose their energy in $\sim 1.9 \times 10^{7} \mathrm{yr}$, which, from equation (24), corresponds to a propagation length of $\sim 2.4 \mathrm{kpc}$. As this distance is consistent with the maximum values measured for our sample, we conclude that it takes a galaxy $\sim 5 \times 10^{7}$ yr to completely traverse this region if it stopped forming stars after a single episode and all Type II SNe occur on timescales $\lesssim 3 \times 10^{7} \mathrm{yr}$. This is, of course, a lower limit, since galaxies have numerous star formation sites with a range of ages in their disks and star formation in spirals never completely turns off.

3. FIR heating by an old stellar population?-Galaxies in this region are characterized by low star formation activity and have best-fit scale lengths that are shorter than expected given the trend found in region II. While we expect the best-fit scale lengths to reach some fixed value for galaxies having constant SFRs over timescales comparable to, or longer than, the CR electron cooling timescale and not increase indefinitely with de- creasing infrared surface brightness, several possible explanations can be considered for the observed turnover.

Let us assume that this is not a signal-to-noise ratio effect. One way to explain the shorter than expected scale lengths is with an increase of CR electron escape. If this were the case, we would then expect to find global FIR/radio ratios that are systematically higher than those for the more active star-forming galaxies. Alternatively, this observation could be explained if the less active star-forming galaxies have larger thermal radio fractions than the higher surface brightness galaxies. In this scenario, we would instead expect to find global FIR/radio ratios that are systematically lower than what is observed for the galaxies with higher radiation field energy densities.

A nonlinearity is in fact observed for the global FIR-radio correlation; galaxies having $L_{\mathrm{FIR}} \lesssim 10^{9} L_{\odot}$ have radio luminosities that are lower than what their FIR luminosities would predict (Condon 1992). In Figure $12 a$ we plot the global $q_{\text {FIR }}$ ratios versus FIR luminosity for the entire WSRT-SINGS sample. The known nonlinearity is clearly observed. In Figure $12 b$ we instead plot $q_{\text {FIR }}$ against $U_{\text {rad }}$; here we do not see any evidence for the lowest surface brightness galaxies to exhibit systematically larger or lower FIR/radio ratios. It then seems that the above explanations (i.e., increased $\mathrm{CR}$ electron escape or larger thermal radio fractions) are not able to reconcile the short scale lengths found for objects in region III.

We also note that the trend found between the best-fit structure scale lengths and radiation field energy density does not exhibit a turnover from regions II to III as seen for best-fit disk and global scale lengths. This suggests that the propagation of $\mathrm{CR}$ electrons associated with star-forming complexes is largely similar despite the differences in the galaxy's global star formation activity. Then, to simultaneously explain this result from our two-component analysis along with the depressed best-fit global scale lengths, we may consider a situation in which older stars contribute significantly to the FIR heating. This will make the FIR emission appear more diffuse and require less smoothing to best match the distribution of radio emission, as observed.

4. Unpopulated part of phase space.-We find no galaxies occupying this region of phase space in which relatively short global scale lengths would be measured for moderate values of $U_{\mathrm{rad}}$. One way for a galaxy to populate this part of the diagram is if, after a long period of quiescence, it begins to form stars at a moderate rate. The galaxy would then pass through this region very quickly $\left(\$ 10^{7} \mathrm{yr}\right)$ before shifting into region II. The lack of such galaxies suggests that, at least in the local universe, star formation in spirals does not completely cease for long periods of time. 
5. CR electron escape.-As stated in $\S 6.1$, the irregulars behave markedly different than the sample spirals. We do not believe this discrepant behavior to be the result of signal-to-noise ratio effects. However, we do note that the radiation field energy densities of these galaxies, as derived from their infrared surface brightnesses alone, may be significantly underestimated due to the low dust content and more transparent media in irregular galaxies. While shifting these objects to larger values of $U_{\text {rad }}$ in Figure 11, they would still clearly remain outliers compared to the rest of the sample. The morphologies and ISM of these galaxies do not seem consistent with keeping their CR electrons bound outside of their initial clouds around SNRs. After the CR electrons leave this cloud, the lack of a dense ISM and magnetic field to keep them trapped through multiple scatterings off of magnetohydrodynamic (MHD) waves allows them to easily escape the system and enter intergalactic space.

Evidence for this picture comes from the extremely short bestfit scale lengths measured for these galaxies. The inability to retain CR electrons once they leave discrete star-forming regions should lead to deviations in the FIR-radio correlation; ratios should be significantly higher than the nominal value, which is in fact observed. In Figure $12 c$ we plot the global FIR/radio ratio as a function of morphological type. All three irregular galaxies in our sample clearly exhibit FIR/radio ratios that are higher than the average value for the entire sample; the average $q_{\text {FIR }}$ for the irregulars is $\sim 0.34$ dex larger than the mean for the entire sample. Furthermore, detailed multiwavelength studies of the SINGS dwarf galaxies have uncovered these deviations within individual systems as well. Strong variations $(\sim 1 \mathrm{dex})$ have been found in the TIR/radio ratio of IC 2574 (Cannon et al. 2005), while the radio continuum emission in the dwarf starburst NGC 1705 has been found to be an order of magnitude less than what one would expect from the nominal TIR/radio ratio (Cannon et al. 2006). A more detailed, systematic study of the FIR and radio properties for dwarf galaxies is beyond the scope of this paper.

\section{REMAINING ISSUES AND FUTURE PROSPECTS}

At the present, we appear to be reaching the limitations on what we can learn about the evolution of CR electrons in nearby galaxies with current data. Using more reliable SFR tracers (e.g., $24 \mu \mathrm{m}+\mathrm{H} \alpha$; Calzetti et al. 2007; Kennicutt et al. 2007) to compare with the radio should improve our ability to understand the effects discussed in $\S$ 7.3. Modeling is also needed and should lead to quantitative estimates for the SFR variations from the placement of a galaxy in Figure 11; this can be a powerful tool for constraining the recent star formation histories of these galaxies.

Due to our small sample size, especially for the lowest surface brightness galaxies, a number of outstanding issues remain. For one, it is currently difficult to determine whether the turnover seen in Figure 11 is in fact physically driven or simply due to a signal-to-noise ratio effect; deeper integrations are clearly needed. At the very high surface brightness end of the spectrum there is even less information to gauge how well our proposed phenomenology applies since the present sample lacks any starbursting luminous infrared galaxies (LIRGs); such galaxies are typically too distant to resolve on $\lesssim 1 \mathrm{kpc}$ scales with current FIR telescopes. We speculate that such galaxies will behave similarly to the high surface brightness galaxies in our sample, although strong galactic winds arising from the central starburst could complicate the picture by transporting CR electrons directly out of the disk or perturbing severely the magnetic field structure. The fact that such galaxies obey the FIR-radio correlation sug- gests that the magnetic field must be amplified for synchrotron cooling to retain the same fraction of CR electron energy losses relative to escape and other competing processes. It is only with additional observations that these outstanding issues can be addressed.

Applying the smoothing phenomenology to such galaxies will add important new insight on the role of magnetic fields and CRs in the process of star formation and galaxy evolution as a whole; starbursting ultraluminous galaxies contribute significantly to the luminosity density of the universe during the most critical epochs $(z \gtrsim 1)$ of galaxy evolution. More than $90 \%$ of UV and visible radiation from starbursting ULIRGs is absorbed by dust, leaving FIR and radio observations as the most promising way to gain insight on the star formation processes within such systems and better understand the physical connection between the gaseous and relativistic phases of the ISM. Such a connection includes that of feedback processes; the production and expulsion of CRs in galaxies may work to cap their luminosity and star formation intensity (Socrates et al. 2008). The outlook for such additional observations appears rather bright; with Herschel and the EVLA on the horizon, increasing the sample size and improving the sensitivity and resolution of the observations, allowing the inclusion of more distant galaxies, should become achievable within a few years.

\section{SUMMARY AND CONCLUSIONS}

Using a two-component image-smearing analysis, we have separated the signatures of CR electron diffusion at spatial scales corresponding to star-forming structures $(<1 \mathrm{kpc})$ and galaxy disks ( $\geq 1 \mathrm{kpc}$ ) within 18 galaxies observed as part of SINGS and WSRT-SINGS. Our results and conclusions can be summarized as follows:

1. We confirm and extend earlier results of M06a and M06b. Empirically, the dispersion in the FIR-radio correlation within galaxies is most reduced by an image-smearing model; this improvement is significantly better than what can be achieved by fitting correlations and removing linear trends.

2. The best-fit global scale lengths decrease as a function of increasing star formation activity as measured by the infrared surface brightness of a galaxy. Our interpretation is that a galaxy's CR electrons are closer to their place of origin within galaxies having intense star formation activity.

3. The trend of decreasing best-fit global scale length with increasing radiation field energy density is due to higher surface brightness galaxies having undergone a recent enhancement of star formation rather than variations in other ISM parameters. For sufficiently large enhancements, these galaxies are observed within $\sim 10^{8} \mathrm{yr}$ of the onset of the most recent star formation episode.

4. Unlike spirals, irregular galaxies lack any well-defined diffuse disk component at either $70 \mu \mathrm{m}$ or especially $22 \mathrm{~cm}$. Presumably, the CR electrons escape these galaxies soon after leaving their parent star-forming regions due to the absence of a dense ISM, which would keep large-scale interstellar magnetic field locked into place. This conclusion helps to explain why these galaxies have global FIR/radio ratios systematically greater than canonical values.

5. As infrared surface brightness increases, the characteristic diffusion scale length of a galaxy's CR electron population begins to transition at $\log U_{\text {rad }} \leq-12.5$, or $\log \Sigma_{\text {SFR }} \leq-2.3$, from being biased by $\mathrm{CR}$ electrons making up its diffuse disk to being biased by those recently injected near star-forming structures. From this we conclude that a galaxy's CR electron population 
transitions from being dominated by old CR electrons to being dominated by young $\mathrm{CR}$ electrons as a function of star formation intensity.

6. The two-component analysis works better than smearing with a single smoothing kernel for spiral galaxies of type $\mathrm{Sb}$ or later, which have high amounts of ongoing star formation activity (i.e., $\sim 40 \%$ of the sample). This result suggests that star formation must be intense and highly structured for the twocomponent analysis of these data to differentiate properly between the different CR electron populations.

We wish to thank the referee, Don Cox, for his insightful comments, which have greatly improved the paper. E. J. M. is indebted to Eric Slezak for his highly constructive comments and critical remarks that helped guide the technical aspects of the wavelet decomposition. E. J. M. would also like to thank other members of the Spitzer Infrared Nearby Galaxies Survey (SINGS) team for their invaluable contributions to the work presented here, especially those of R. C. Kennicutt Jr., D. Calzetti, K. D. Gordon, C. W. Engelbracht, and G. Bendo. As part of the Spitzer Space Telescope Legacy Science Program, support was provided by NASA through contract 1224769 issued by the Jet Propulsion Laboratory, California Institute of Technology under NASA contract 1407. E. J. M. also acknowledges support for this work provided by the Spitzer Science Center Visiting Graduate Student program. This research has made use of the NASA/IPAC Extragalactic Database, which is operated by JPL/Caltech, under contract with NASA. This publication makes use of data products from the Two Micron All Sky Survey, which is a joint project of the University of Massachusetts and IPAC/Caltech, funded by NASA and the National Science Foundation.
Adami, C., et al. 2005, A\&A, 429, 39

Ball, R., Sargent, A. I., Scoville, N. Z., Lo, K. Y., \& Scott, S. L. 1985, ApJ, 298, L21

Beck, R. 2005, in Cosmic Magnetic Fields, ed. R. Wielebinsky \& R. Beck (Berlin: Springer), 41

. 2007, A\&A, 470, 539

Beck, R., Brandenburg, A., Moss, D., Shukurov, A., \& Sokoloff, D. 1996, ARA\&A, 34, 155

Beck, R., \& Golla, G. 1988, A\&A, 191, L9

Bell, A. R. 1978, MNRAS, 182, 147

Bell, E. F. 2003, ApJ, 586, 794

Berezhko, E. G., Ksenofontov, L. T., Ptuskin, V. S., Zirakashvili, V. N., \& Völk, H. J. 2003, A\&A, 410, 189

Bicay, M. D., \& Helou, G. 1990, ApJ, 362, 59

Bijaoui, A. 1991, in Ondelettes et Paquets d'Ondes, ed. P. J. Lions (Proc. Cours CEA/EdF/INRIA), 115

Bijaoui, A., \& Rué, F. 1995, Signal Proc., 46, 229

Blandford, R. D., \& Ostriker, J. P. 1978, ApJ, 221, L29

Bloemen, J. B. G. M., et al. 1986, A\&A, 154, 25

Boulares, A., \& Cox, D. P. 1990, ApJ, 365, 544

Braun, R., Oosterloo, T. A., Morganti, R., Klein, U., \& Beck, R. 2007, A\&A, 461,455

Bronkalla, W., Notni, P., \& Mutter, A. A.-R. 1992, Astron. Nachr., 313, 1

Calzetti, D., et al. 2005, ApJ, 633, 871

2007, ApJ, 666, 870

Cannon, J. M., et al. 2005, ApJ, 630, L37 2006, ApJ, 647, 293

Condon, J. J. 1992, ARA\&A, 30, 575

Cox, D. P. 2005, ARA\&A, 43, 337

Dahlem, M., Lisenfeld, U., \& Golla, G. 1995, ApJ, 444, 119

Dale, D. A., Giovanelli, R., Haynes, M. P., Scodeggio, M., Hardy, E., \& Campusano, L. E. 1997, AJ, 114, 455

Dale, D. A., \& Helou, G. 2002, ApJ, 576, 159

Dale, D. A., et al. 2007, ApJ, 655, 863

de Jong, T., Klein, U., Wielebinski, R., \& Wunderlich, E. 1985, A\&A, 147, L6 de Vaucouleurs, G., de Vaucouleurs, A., Corwin, H. G., Jr., Buta, R. J., Paturel, G., \& Fouque, P. 1991, Third Reference Catalogue of Bright Galaxies (New York: Springer)

Dove, J. B., \& Shull, J. M. 1994, ApJ, 430, 222

Duric, N. 1991, in ASP Conf. Ser. 18, The Interpretation of Modern Synthesis Observations of Spiral Galaxies, ed. N. Duric \& P. Crane (San Francisco: ASP), 17

Elmegreen, B. G. 1982, ApJ, 253, 655

Ferguson, A. M. N., Wyse, R. F. G., Gallagher, J. S., \& Hunter, D. A. 1996, AJ, 111,2265

Ferrarese, L., et al. 2000, ApJ, 529, 745

Ferrière, K. 2001, Rev. Mod. Phys., 73, 1031

Fletcher, A., Beck, R., Berkhuijsen, E. M., Horellou, C., \& Shukurov, A. 2004, in How Does the Galaxy Work?, ed. E. J. Alfaro et al. (Dordrecht: Kluwer), 29

Freedman, W. L., et al. 2001, ApJ, 553, 47

Frick, P., Beck, R., Berkhuijsen, E. M., \& Patrickeyev, I. 2001, MNRAS, 327, 1145

Gil de Paz, A., et al. 2007, ApJS, 173, 185

Ginzburg, V. L., Khazan, Ya. M., \& Ptuskin, V. S. 1980, Ap\&SS, 68, 295
Gordon, K. D., et al. 2005, PASP, 117, 503

Haslam, C. G. T., Stoffel, H., Salter, C. J., \& Wilson, W. E. 1982, A\&AS, 47, 1

Helou, G., \& Bicay, M. D. 1993, ApJ, 415, 93

Helou, G., Soifer, B. T., \& Rowan-Robinson, M. 1985, ApJ, 298, L7

Hippelein, H., Haas, M., Tuffs, R. J., Lemke, D., Stickel, M., Klaas, U., \& Völk, H. J. 2003, A\&A, 407, 137

Hoernes, P., Berkhuijsen, E. M., \& Xu, C. 1998, A\&A, 334, 57

Holdschneider, M., Kronland-Martinet, R., Morlet, J., \& Tchamitchian, P. 1989, in Wavelets, ed. J. M. Combes, A. Grossmann, \& P. Tchamitchian (Berlin: Springer), 286

Hughes, A., Wong, T., Ekers, R., Staveley-Smith, L., Filipovic, M., Maddison, S., Fukui, Y., \& Mizuno, N. 2006, MNRAS, 370, 363

Hummel, E., Lesch, H., Wielebinski, R., \& Schlickeiser, R. 1988, A\&A, 197, L29

Irwin, J. A., English, J., \& Sorathia, B. 1999, AJ, 117, 2102

Jarrett, T. H., Chester, T., Cutri, T., Schneider, S., \& Huchra, J. 2003, AJ, 125, 525

Jones, F. C., Lukasiak, A., Ptuskin, V., \& Webber, W. 2001, ApJ, 547, 264

Karachentsev, I. D., Sharina, M. E., \& Huchtmeier, W. K. 2000, A\&A, 362, 544

Karachentsev, I. D., et al. 2002, A\&A, 383, 125 2003, A\&A, 398, 479

Kennicutt, R. C., Jr., et al. 2003, PASP, 115, 928 2007, ApJ, 671, 333

Leonard, D. C., et al. 2002, AJ, 124, 2490

Lisenfeld, U., Alexander, P., Pooley, G. G., \& Wilding, T. 1996a, MNRAS, 281,301

Lisenfeld, U., Völk, H. J., \& Xu, C. 1996b, A\&A, 306, 677

Macri, L. M., Stetson, P. B., Bothun, G. D., Freedman, W. L., Garnavich, P. M.,

Jha, S., Madore, B. F., \& Richmond, M. W. 2001, ApJ, 559, 243

Marsh, K. A., \& Helou, G. 1995, ApJ, 445, 599

. 1998, ApJ, 493, 121

Martin, D. C., et al. 2005, ApJ, 619, L1

Masters, K. 2005, Ph.D. thesis, Cornell Univ.

Maurin, D., Donato, F., Taillet, R., \& Salati, P. 2002, A\&A, 394, 1039

McKee, C. F. 1995, in ASP Conf. Ser. 80, The Physics of the Interstellar Medium and Intergalactic Medium, ed. A. Ferrara et al. (San Francisco: ASP), 292

Moskalenko, I. V., Strong, A. W., Ormes, J. F., \& Potgieter, M. S. 2002, ApJ, 565,280

Mouschovias, T. Ch., Shu, F. H., \& Woodward, P. R. 1974, A\&A, 33, 73

Murphy, E. J., et al. 2006a, ApJ, 638, 157 (M06a) 2006b, ApJ, 651, L111 (M06b)

Niklas, S. 1995, Ph.D. thesis, Univ. Bonn 1997, A\&A, 322, 29

Niklas, S., \& Beck, R. 1997, A\&A, 320, 54

Niklas, S., Klein, U., \& Wielebinski, R. 1997, A\&A, 322, 19

Osterbrock, D. E. 1989, Astrophysics of Gaseous Nebulae and Active Galactic Nuclei (Mill Valley: University Science Books)

Parker, E. N. 1966, ApJ, 145, 811

Pérez-González, P. G., et al. 2006, ApJ, 648, 987

Prescott, M. K. M., et al. 2007, ApJ, 668, 182

Rebusco, P., Churazov, E., Böhringer, H., \& Forman, W. 2005, MNRAS, 359, 1041

Rieke, G. H., et al. 2004, ApJS, 154, 25 
Roediger, E., Brüggen, M., Rebusco, P., Böhringer, H., \& Churazov, E. 2007, MNRAS, 375,15

Rubin, R. H. 1968, ApJ, 154, 391

Rué, F., \& Bijaoui, A. 1997, Exp. Astron., 7, 129

Ruzmaikin, A. A., Sokoloff, D. D., \& Shukurov, A. M. 1988, Nature, 336, 341

Schlegel, D. J., Finkbeiner, D. P., \& Davis, M. 1998, ApJ, 500, 525

Seth, A. C., Dalcanton, J. J., \& de Jong, R. S. 2005, AJ, 129, 1331

Shapley, A., Fabbiano, G., \& Eskridge, P. B. 2001, ApJS, 137, 139

Socrates, A., Davis, S. W., \& Ramirez-Ruiz, E. 2008, ApJ, in press (astro-ph/ 0609796)

Starck, J.-L., Bijaoui, A., Valtchanov, I., \& Murtagh, F. 2000, A\&AS, 147, 139

Starck, J.-L., \& Murtagh, F. 2002, Astronomical Image and Data Analysis (Berlin: Springer)

Stil, J. M., \& Israel, F. P. 2002, A\&A, 389, 42

Strong, A. W., \& Moskalenko, I. V. 1998, ApJ, 509, 212
Strong, A. W., Moskalenko, I. V., \& Ptuskin, V. S. 2007, Annu. Rev. Nucl. Part. Sci., 57,285

Strong, A. W., Moskalenko, I. V., \& Reimer, O. 2000, ApJ, 537, 763

Tabatabaei, F. S., et al. 2007, A\&A, 466, 509

Thompson, T. A., Quataert, E., Waxman, E., Murray, N., \& Martin, C. L. 2006, ApJ, 645, 186

Tonry, J. L., Dressler, A., Blakeslee, J. P., Ajhar, E. A., Fletcher, A. B., Luppino, G. A., Metzger, M. R., \& Moore, C. B. 2001, ApJ, 546, 681

Tully, R. B. 1988, Nearby Galaxies Catalog (Cambridge: Cambridge Univ. Press) Webber, W. R. 1991, in ASP Conf. Ser. 18, The Interpretation of Modern Synthesis Observations of Spiral Galaxies, ed. N. Duric \& P. Crane (San Francisco: ASP), 27

Xu, C., Klein, U., Meinert, D., Wielebinski, R., \& Haynes, R. F. 1992, A\&A, 257, 47

Yun, M. S., Reddy, N. A., \& Condon, J. J. 2001, ApJ, 554, 803 